\title{
Analysis of a High-Dimensional Mathematical Model for Plant Virus Transmission with Continuous and Impulsive Roguing Control
}

\author{
Guangming Qiu $\mathbb{D}^{1,2}$ Sanyi Tang ${ }^{1}{ }^{1}$, and Mengqi He ${ }^{1}{ }^{1}$ \\ ${ }^{1}$ School of Mathematics and Statistics, Shaanxi Normal University, Xi'an, Shaanxi 710062, China \\ ${ }^{2}$ School of Mathematics and Statistics, Qinghai Normal University, Xining, Qinghai 810016, China \\ Correspondence should be addressed to Sanyi Tang; sytang@snnu.edu.cn
}

Received 11 October 2021; Accepted 22 November 2021; Published 14 December 2021

Academic Editor: Binxiang Dai

Copyright (c) 2021 Guangming Qiu et al. This is an open access article distributed under the Creative Commons Attribution License, which permits unrestricted use, distribution, and reproduction in any medium, provided the original work is properly cited.

\begin{abstract}
Roguing and replanting are the most common strategies to control plant diseases and pests. How to build the mathematical models of plant virus transmission and consider the impact of roguing and replanting strategies on plant virus eradication is of great practical significance. In the present paper, we propose the mathematical models for plant virus transmission with continuous and impulsive roguing control. For the model with continuous control strategies, the threshold values for the existences and stabilities of multiple equilibria have been given, and the effect of roguing strategies on the threshold values is also addressed. Furthermore, the model with impulsive roguing control tactics is proposed, and the existence and stability of the plantonly and disease-free periodic solutions of the model are investigated by calculating several threshold values. Moreover, when selecting the design control strategy to minimize the threshold, we systematically analyze the existence of the optimal times of roguing infected plants within a replanting cycle, which is of great significance to the design and optimization of the prevention and control strategy of plant virus transmission. Finally, numerical investigations are given to reveal the main conclusions, and the biological implications of the main results are briefly discussed in the last section.
\end{abstract}

\section{Introduction}

Plant diseases are caused by plant viruses transmitted by insect vectors [1-5], which can cause serious losses in economy and increased poverty [6]. Moreover, vector activity and behavior, especially in relation to virus transmission, are key to determining severity of plant disease transmission [7-13]. Therefore, the development of corresponding prevention and control strategies of plant infectious diseases plays a very important role in protecting plants and eradicating vectors. Note that current control tactics of plant disease mainly include gene resistance, vector insect, and culture control. Gene resistance control cannot be widely used due to its high cost, and vector control through insecticide spraying has a small risk of failure due to selection for more damaging virus strains [14-16]. However, the cultural control including roguing or removal of diseased plants has been practised and could be more effective [17-20]. Therefore, a successful plant disease control program depends on a plant production system which is closely aligned with the goals of pest management [21-23].

How to evaluate the effectiveness and timeliness of these prevention and control measures needs the development of corresponding mathematical models. A large number of studies have proved that mathematical modeling approaches can help in the prediction of disease propagation in the agricultural realm $[8,13,22,24-27]$. More recently, several mathematical models for the spread of plant diseases in populations have been analyzed mathematically and applied to control plant diseases. For example, a deterministic model with bilinear incidence was formulated to address the impact of cross protection on the spread and control of Huanglongbing (HLB) disease [22], and 
the main results reveal that cross protection and removing infected trees play an important role in controlling the spread of HLD. In literature [24], the authors developed an individualbased model (IBM) of vector-borne plant pathogen spread and compared the simulation results with a classical continuous model. In [25], the authors proposed a model with time delay to study the effect of both incubation delay and latent time on the dynamics of plant disease. A more realistic model has been developed in [26] which takes within-plant cell processes, vector population dynamics, behavior, and broader ecological interactions into account. In [27], considering awareness as a controlling measure, the effect of time delay and awareness campaign on the dynamics and control of the mosaic disease has been investigated.

Recently, impulsive differential equations have been employed as the mathematical models for many real problems including pest control [16, 28-30], infectious diseases control, and drug administration [31]. Moreover, various plant disease models with impulsive control have been developed and studied [17-20, 32-34]. In [17], the authors proposed a mathematical model for plant population which aims to eradicate infected plants or maintain the number of infected plants below the economic threshold. In $[18,19]$, the plant disease models with impulsive cultural strategies were developed and analyzed, and the main results reveal that the plant disease could be eradicated or the number of infected plants could be maintained below the economic threshold by choosing proper control frequency and intensity. We note that those modelling methods have been developed and extended in various references [20, 32-34].
However, most of the above works only consider (a) the transmission of diseases among a single plant population, without considering the effect of the vector population on the disease transmission; (b) the continuous human intervention strategies, without considering the instantaneity of intervention strategies; and (c) the impact of pulse control on infected plants, without considering the inevitable impact of control measures on healthy plants. Considering the above shortcomings, this paper aims to develop mathematical models, fully integrate the above three factors, develop corresponding theoretical analysis and numerical techniques, and systematically and deeply analyze their impact on plant disease control. In particular, various threshold values which guarantee the existence and stability of plant-only and disease-free periodic solutions have been obtained, and the existence of the optimal times of roguing infected plants within a replanting cycle has been also investigated in more detail.

\section{The Model}

2.1. System Description. In this section, based on the model proposed in [21], we develop a vector-borne plant disease model with cultural control. To do this, we denote the total plant population at time $t$ as $N(t)=X(t)+Y(t)$ and vector population as $M(t)=U(t)+V(t)$. The population flow among those compartments is shown in the schematic diagram (i.e., Figure 1), which leads to the following model:

$$
\left\{\begin{array}{l}
\frac{\mathrm{d} X(t)}{\mathrm{d} t}=r-k_{1} X(t) V(t)-g X(t), \\
\frac{\mathrm{d} Y(t)}{\mathrm{d} t}=k_{1} X(t) V(t)-g Y(t)-\alpha Y(t), \\
\frac{\mathrm{d} U(t)}{\mathrm{d} t}=b(U(t)+V(T))\left(1-\frac{U(t)+V(t)}{m(X(t)+Y(t))}\right)-k_{2} U(t) Y(t)-c U(t), \\
\frac{\mathrm{d} V(t)}{d \mathrm{t}}=k_{2} U(t) Y(t)-c V(t),
\end{array}\right.
$$

where $X(t), Y(t), U(t)$, and $V(t)$ represent the number of susceptible plants, infectious plants, susceptible vectors, and infectious vectors, respectively. The notations and definitions of all parameters are shown in Table 1 .
2.2. Basic Properties. In this section, we show the boundedness of system (1), and it follows from system (1) that we have

$$
\frac{\mathrm{d} N(t)}{\mathrm{d} t}=r-g X(t)-(\alpha+g)(t) \leq r-g N(t),
$$

which indicates

$$
N(t) \leq N(0) \exp (-g t)+\frac{r}{g}(1-\exp (-g t)) \longrightarrow \frac{r}{g}, \quad \text { for } t \longrightarrow \infty
$$




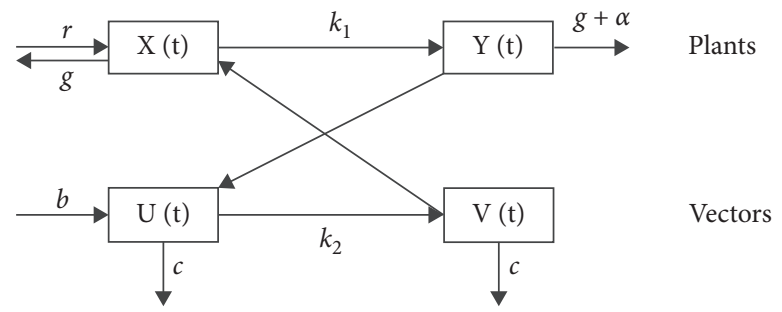

Figure 1: The transfer diagram for model (1).

TABle 1: Definitions of all parameters and their baseline values.

\begin{tabular}{|c|c|c|c|c|}
\hline Parameter & Interpretation & Standard value & Range & Source \\
\hline$r$ & Replanting rate & $0.005\left(\right.$ day $\left.^{-1}\right)$ & $0-0.025$ & {$[21]$} \\
\hline$\alpha$ & Plant roguing rate & $0.003\left(\right.$ day $\left.^{-1}\right)$ & $0-0.033$ & {$[21]$} \\
\hline$g$ & Plant loss/harvesting rate & $0.003\left(\right.$ day $\left.^{-1}\right)$ & $0.002-0.004$ & [21] \\
\hline$m$ & Maximum vector abundance & $500\left(\right.$ plant $\left.^{-1}\right)$ & $0-2500$ & {$[21]$} \\
\hline$b$ & Maximum vector birth rate & $0.2\left(\mathrm{day}^{-1}\right)$ & $0.1-0.3$ & {$[21]$} \\
\hline$c$ & Vector mortality & $0.12\left(\right.$ day $\left.^{-1}\right)$ & $0.06-0.18$ & {$[21]$} \\
\hline$k_{1}$ & Infection rate & $0.008\left(\right.$ vector $^{-1}$ day $\left.^{-1}\right)$ & $0.002-0.032$ & [21] \\
\hline$k_{2}$ & Acquisition rate & $0.008\left(\right.$ plant $^{-1}$ day $\left.^{-1}\right)$ & $0.002-0.032$ & [21] \\
\hline
\end{tabular}

Similarly, we have

$$
\frac{\mathrm{d} M(t)}{\mathrm{d} t}=b M(t)\left(1-\frac{M(t)}{m N(t)}\right)-c M(t) \leq(b-c) M(t)-\frac{b g M^{2}(t)}{m r}
$$

and consequently we get

$$
M(t) \leq \frac{M(0) \exp ((b-c) t)}{1+b g M(0)(\exp ((b-c) t)-1) / m r(b-c)} \longrightarrow \frac{m r(b-c)}{b g}, \text { for } t \longrightarrow \infty
$$

Thus, the total plant population $N(t)$ and vector population $M(t)$ are uniformly bounded. Furthermore, the region

$$
\Omega=\left\{(X(t), Y(t), U(t), V(t)) \in R_{+}^{4}: X(t)+Y(t) \leq \frac{r}{g} ; U(t)+V(t) \leq \frac{m r(b-c)}{b g}\right\}
$$

is positively invariant, which indicates that we can focus on the dynamics of system (1) on the set $\Omega$ in the following.

\section{Dynamics of the Model with Continuous Control}

3.1. Boundary Equilibria and Their Stabilities. System (1) has plant-only equilibrium $E_{01}=\left(X_{0}, 0,0,0\right)=(r / g, 0,0,0)$ and disease-free equilibrium $E_{02}=\left(X_{0}, 0, U_{0}, 0\right)=(r / g, 0, m r$ $(b-c) / b g, 0)$ with $b>c$. By employing the next generation matrix method [35], we can obtain the basic reproduction number of model (1) as follows:

$$
R_{0}=R_{01} R_{02}=\sqrt{\frac{k_{1} k_{2} m r^{2}(b-c)}{b c g^{2}(\alpha+g)}},
$$

where $R_{01}=\sqrt{k_{1} r / c g}, R_{02}=\sqrt{k_{2} m r(b-c) / b g(\alpha+g)} . \quad R_{0}$ represents the number of secondary cases that one infected individual will cause through the duration of the infective period, $R_{01}$ is the expected number of plants that one vector 
infects throughout its infectious life time, and $R_{02}$ is the expected number of vectors that one plant infects throughout its infectious life time.

Theorem 1. The plant-only equilibrium $E_{01}$ is globally asymptotically stable if $b \leq c$ and $R_{01} \leq 1$.
Proof. We introduce the following Lyapunov function:

$$
V_{1}(t)=X-X_{0}-X_{0} \ln \frac{X}{X_{0}}+Y+M+V,
$$

and further

$$
\begin{aligned}
\dot{V}_{1}(t) & =\left(1-\frac{X_{0}}{X}\right) X\left(r\left(\frac{1}{X}-\frac{1}{X_{0}}\right)-k_{1} V\right)+k_{1} X V-(\alpha+g) Y+(b-c) M-\frac{b M^{2}}{m(X+Y)}+k_{2} U Y-c V \\
& =r\left(2-\frac{X}{X_{0}}-\frac{X_{0}}{X}\right)+\left(k_{1} X_{0}-c\right) V+\left(k_{2} U-\alpha+g\right) Y+(b-c) M-\frac{b M^{2}}{m(X+Y)} \\
& \leq r\left(2-\frac{X_{0}}{X}-\frac{X}{X_{0}}\right)+c\left(R_{01}^{2}-1\right) V+\left(k_{2} M-\alpha+g\right) Y+(b-c) M-\frac{b M^{2}}{m(X+Y)} \\
& \leq r\left(2-\frac{X_{0}}{X}-\frac{X}{X_{0}}\right)+c\left(R_{01}^{2}-1\right) V+(\alpha+g)\left(\frac{k_{2} m r(b-c)}{(\alpha+g) b g}-1\right) Y+(b-c) M-\frac{b M^{2}}{m(X+Y)}
\end{aligned}
$$

The arithmetical mean is great than or equal to the geometrical mean, and the function $2-\left(X_{0} / X\right)-\left(X / X_{0}\right)$ is nonpositive for all $X>0$, and it is equal to zero if and only if $X=X_{0}$. If $b \leq c$ and $R_{01} \leq 1$, then

$$
r\left(2-\frac{X_{0}}{X}-\frac{X_{0}}{X}\right)+c\left(R_{01}^{2}-1\right) V+(\alpha+g)\left(\frac{k_{2} m r(b-c)}{(\alpha+g) b g}-1\right) Y+(b-c)(M)-\frac{b M^{2}}{m(X+Y)} \leq 0
$$

Thus, we have $\dot{V}_{1} \leq 0$. Furthermore, $\dot{V}_{1}=0$ only if $Y=0$, $U=0$, and $V=0$, which indicates that the maximum invariant set in $\left\{\left(X_{0}, 0,0,0\right): \dot{V}=0\right\}$ is the singleton $E_{01}$. By LaSalle's Invariance Principle [36], we conclude that $E_{01}$ is globally asymptotically stable in $\Omega$.
Next, we show that the disease-free equilibrium is globally stable provided that $R_{0}<1$. First, the Jacobian matrix at the equilibrium $E_{02}$ is given by

$$
J_{E_{02}}=\left(\begin{array}{cccc}
-g & 0 & 0 & \frac{-k_{1} r}{g} \\
0 & -(\alpha+g) & 0 & \frac{k_{1} r}{g} \\
\frac{m(b-c)^{2}}{b} \frac{m(b-c)^{2}}{b}-\frac{k_{2} m r(b-c)}{b g} & -(b-c) & 2 c-b \\
0 & \frac{k_{2} m r(b-c)}{b g} & 0 & -c
\end{array}\right) .
$$


Stability of equilibrium $E_{02}$ is determined by the eigenvalues of the Jacobian $J_{E_{02}}$ evaluated at that equilibrium. Characteristic equation at disease-free equilibrium $E_{02}$ becomes

$$
\begin{aligned}
& (\lambda+(b-c))(\lambda+g)\left(\lambda^{2}+(\alpha+g+c) \lambda\right. \\
& \left.+c(\alpha+g)\left(1-R_{0}^{2}\right)\right)=0 .
\end{aligned}
$$

It is easy to see that two roots are $\lambda_{1}=-(b-c)<0$ and $\lambda_{2}=-g<0$, and according to Routh-Hurwitz condition, $\lambda^{2}+$ $(\alpha+g+c) \lambda+c(\alpha+g)\left(1-R_{0}^{2}\right)=0$ has roots with negative real parts when $R_{0}<1$. Therefore, $E_{02}$ is stable if $R_{0}<1$. Furthermore, we have the following result for the global stability of $E_{02}$.
Theorem 2. For system (1), if $R_{0} \leq 1$, then the disease-free equilibrium $E_{02}$ is globally asymptotically stable.

Proof. Define a Lyapunov function:

$$
V_{2}(t)=\frac{k_{2} m r c g(b-c)}{2 k_{1} r} Y^{2}+\frac{b g(g+\alpha)}{2} V^{2}+k_{2} m r(b-c) Y V
$$

and then the derivative of $V_{2}(t)$ with respect to system (1) is given by

$$
\begin{aligned}
\dot{V}_{2}(t)= & \frac{k_{2} m r c g(b-c)}{k_{1} r} Y\left(k_{1} X V-(g+\alpha) Y\right)+b g(g+\alpha) V\left(k_{2} U Y-c V\right) \\
& +b g(g+\alpha)\left(k_{1} X V-(g+\alpha) Y\right)+b g(g+\alpha) Y\left(k_{2} U Y-c V\right) \\
= & \left(k_{2} m c g(b-c) X+k_{2} b g(g+\alpha) U-k_{2} m r(b-c)(g+\alpha)-k_{2} m r(b-c) c\right) Y V \\
& +\left(k_{2}^{2} m r(b-c) U-\frac{k_{2} m r c g(b-c)(g+\alpha)}{k_{1} r}\right) Y^{2}+\left(k_{1} k_{2} m r(b-c) X-c b g(g+\alpha)\right) \\
\leq & \frac{k_{2} m r c g(b-c)(g+\alpha)}{k_{1} r}\left(R_{0}^{2}-1\right) Y^{2}+b c g(g+\alpha)\left(R_{0}^{2}-1\right) V^{2} .
\end{aligned}
$$

Therefore, we have $\dot{V}_{2}(t) \leq 0$ if $R_{0} \leq 1$, and $\dot{V}_{2}(t)=0$ if $R_{0}=1$ or $Y=0, V=0$. Substituting $Y=0, V=0$ into the first and third equations of (1), one has $\dot{X}(t)=r-g X(t)$ and $\dot{U}(t)=b U(t)(1-U(t) / m X(t))-c U(t)$, which implies $X(t) \longrightarrow r / g$ and $U(t) \longrightarrow m r(b-c) / b g$ as $t \longrightarrow+\infty$. Again, it follows from LaSalle's Invariance Principle [36] that $E_{02}$ is globally asymptotically stable in $\Omega$.

3.2. The Existence of Endemic Equilibrium and Uniform Persistence. In order to determine the existence of the endemic equilibrium, we have to look for the solution of the algebraic system of equations obtained by equating the right sides of system (1) to zero.

$$
\begin{aligned}
r-k_{1} X^{*} V^{*}-g X^{*} & =0, \\
k_{1} X^{*} V^{*}-g Y^{*}-\alpha Y^{*} & =0, \\
b\left(U^{*}+V^{*}\right)\left(1-\frac{U^{*}+V^{*}}{m\left(X^{*}+Y^{*}\right)}\right)-k_{2} U^{*} Y^{*}-c U^{*} & =0, \\
k_{2} U^{*} Y^{*}-c V^{*} & =0 .
\end{aligned}
$$

From the first and second equations of (15), we have

$$
\begin{aligned}
X^{*} & =\frac{r}{k_{1} V^{*}+g}, \\
Y^{*} & =\frac{k_{1} r V^{*}}{(\alpha+g)\left(k_{1} V^{*}+g\right)}, \\
U^{*} & =\frac{m(b-c)\left(r(\alpha+g)+k_{1} r V^{*}\right)}{b(\alpha+g)\left(k_{1} V^{*}+g\right)}-V^{*} .
\end{aligned}
$$

Substituting the above into the fourth equation of (15), we have

$$
A\left(V^{*}\right)^{2}+B V^{*}+C=0
$$

where

$$
\begin{aligned}
& A=-k_{1}^{2} b(\alpha+g)\left(k_{2} r+c(\alpha+g)\right)<0, \\
& B=k_{1}^{2} k_{2} m r^{2}(b-c)-k_{1} k_{2} b r g(\alpha+g)-2 k_{1} b c g(\alpha+g)^{2}, \\
& C=b c g^{2}(\alpha+g)^{2}\left(R_{0}^{2}-1\right) .
\end{aligned}
$$

Equation (17) may have two roots if $\Delta>0$, denoted by

$$
\begin{aligned}
& V_{1}^{*}=\frac{-B+\sqrt{\Delta}}{2 A}, \\
& V_{2}^{*}=\frac{-B-\sqrt{\Delta}}{2 A},
\end{aligned}
$$


with $\Delta=B^{2}-4 A C$, and we have the following main results.

Theorem 3. For system (1), if $R_{0}>1$, then there exists a unique endemic equilibrium $E^{*}=\left(X^{*}, Y^{*}, U^{*}, V^{*}\right)$; if $R_{0} \leq 1$, then there is no endemic equilibrium.

Next, we will employ the persistent theory developed in literature [37] to show the uniform persistence of system (1). To do this, we let $E$ be a closed positively invariant subset of $\Omega$, on which a continuous flow $\mathscr{F}$ is defined. We denote the restriction $\mathscr{F}$ to $\partial E$ by $\partial \mathscr{F}$ and note that $\partial E$ is in general not positively invariant. Let $N$ be the maximal invariant set of $\partial \mathscr{F}$ on $\partial E$. Suppose that $N$ is a closed invariant set and there exists a cover $\left\{N_{\alpha}\right\}_{\alpha \in A}$ of $N$, where $A$ is a nonempty index set. $N_{\alpha} \subset \partial E, N \subset \cup_{\alpha \in A} N_{\alpha}$, and $\left\{N_{\alpha}\right\}(\alpha \in A)$ are pairwise disjoint closed invariant sets. Furthermore, we propose the following hypotheses and lemmas. $\left(H_{1}\right)$ All $N_{\alpha}$ are isolated invariant sets of the flow $\mathscr{F} .\left(H_{2}\right)\left\{N_{\alpha}\right\}_{\alpha \in A}$ is acyclic; that is, any finite subset of $\left\{N_{\alpha}\right\}_{\alpha \in A}$ does not form a cycle. $\left(H_{3}\right)$ Any compact subset of $\partial E$ contains, at most, finitely many sets of $\left\{N_{\alpha}\right\}_{\alpha \in A}$ [37].

Lemma 1 (see [37]). Let $E$ be a closed positively invariant subset of $\Omega$ on which a continuous flow $\mathscr{F}$ is defined. Suppose there is a constant $\varepsilon>0$ such that $\mathscr{F}$ is point dissipative on $S[\partial E, \varepsilon] \cap E^{0}$ and the assumptions $\left(H_{1}-H_{3}\right)$ hold. Then, the flow $\mathscr{F}$ is uniformly persistent, if and only if $W^{+}\left(N_{\alpha}\right) \cap S$ $[\partial E, \alpha] \cap E^{0}=\phi$ for any $\alpha \in A$, where $W^{+}\left(N_{\alpha}\right)=\{y \in \Omega, \omega$ $\left.(y) \subset N_{\alpha}\right\}$, and $E^{0}$ is interior of set $E$.

By this lemma, we can show the uniform persistence of disease when $R_{0}>1$.

Theorem 4. In system (1), assume that $R_{0}>1$ and the disease is initially present; then, the disease is uniformly persistent; i.e., there is a constant $\rho>0$ such that $\liminf _{t \rightarrow+\infty} Y(t) \geq \rho$, $\liminf _{t \longrightarrow+\infty} V(t) \geq \rho$.

Proof. We set $E=\left\{(X, Y, U, V) \in \mathbf{R}_{+}^{4} \mid 0 \leq X+Y \leq r / g, 0 \leq U+\right.$ $V \leq m r(b-c) / b g\}, \partial E=\{(X, Y, U, V) \in E \mid Y=0, V=0\}$, and we will show that the conditions of Lemma 1 are satisfied. Clearly, $N_{\alpha}=E_{0}=(r / g, 0, m r(b-c) / b g, 0)$ is isolated. Hence, the covering is simply $N=E_{0}$, which is acyclic. Thus, the conditions $\left(H_{1}-H_{3}\right)$ hold. We can also obtain that $\mathscr{F}$ is point dissipative by (6). Now, we show that $W^{+}\left(E_{0}\right) \cap E^{0}=\phi$. Suppose this is not true; then, there exists a solution $(X(t), Y(t)$, $U(t), V(t)) \in E^{0}$ such that $\lim _{t \longrightarrow+\infty} X(t)=r / g, \lim _{t \longrightarrow+\infty}$ $Y(t)=0, \lim _{t \longrightarrow+\infty} U(t)=m r(b-c) / b g, \lim _{t \longrightarrow+\infty} V(t)=0$. For any sufficiently small constant $\varepsilon>0$, there exists a positive constant $T=T(\varepsilon)$ such that $X(t)>r / g-\varepsilon, Y(t)<\varepsilon, U(t)$ $>m r(b-c) / b g-\varepsilon, V(t)<\varepsilon$ for all $t \geq T$. Since $R_{0}=$ $\sqrt{k_{1} k_{2} m r^{2}(b-c) / b c g^{2}(\alpha+g)}>1$, for sufficiently small $\varepsilon$, we have

$$
R_{0}^{\prime}=\sqrt{\frac{k_{1} k_{2}}{c(\alpha+g)}\left(\frac{m r(b-c)}{b g}-\varepsilon\right)\left(\frac{r}{g}-\varepsilon\right)}>1 .
$$

Note that

$$
\begin{aligned}
& \frac{\mathrm{d} Y(t)}{\mathrm{d} t} \geq k_{1}\left(\frac{r}{g}-\varepsilon\right) V(t)-(g+\alpha) Y(t), \\
& \frac{\mathrm{d} V(t)}{\mathrm{d} t} \geq k_{2}\left(\frac{m r(b-c)}{b g}-\varepsilon\right) Y(t)-c V(t), t \geq T .
\end{aligned}
$$

Therefore, if $Y(t), V(t) \longrightarrow 0$, as $t \longrightarrow \infty$, then by a standard comparison argument and the nonnegativity, the solution $n_{Y}(t), n_{V}(t)$ of

$$
\begin{aligned}
& \frac{\mathrm{d} n_{Y}(t)}{\mathrm{d} t}=k_{1}\left(\frac{r}{g}-\varepsilon\right) n_{V}(t)-(\alpha+g) n_{Y}(t), \\
& \frac{\mathrm{d} n_{V}(t)}{\mathrm{d} t}=k_{2}\left(\frac{m r(b-c)}{b g}-\varepsilon\right) n_{Y}(t)-c n_{V}(t), \quad t \geq T,
\end{aligned}
$$

with initial data $n_{Y}(T)=Y(T), n_{V}(T)=V(T)$, converges to $(0,0)$ as well. Thus, $\lim W_{n}(t)=0$, where $W_{n}(t)>0$ is defined by

$$
W_{n}(t)=C_{1} n_{Y}(t)+C_{2} n_{V}(t)
$$

where $C_{1}=k_{2}, C_{2}=\alpha+g / m r(b-c) / b g-\varepsilon$.

The derivative of $W_{n}(t)$ is given by

$$
\frac{\mathrm{d} W_{n}(t)}{\mathrm{d} t}=\frac{c(\alpha+g)}{m r(b-c) / b g-\varepsilon}\left(R_{0}^{\prime 2}-1\right) n_{V}(t) \geq 0 .
$$

Therefore, $W_{n}(t)$ goes to either infinity or some positive number as $t \longrightarrow \infty$, which is a contradiction of $\lim _{t \longrightarrow+\infty} W_{n}(t)=0$. Thus, we have $W^{+}\left(E_{0}\right) \cap E^{0}=\phi$. Then, we obtain $\lim _{t \longrightarrow+\infty} V(t) \geq \rho_{1}$ for some constant $\rho_{1}>0$. By the fourth equation of $(1)$, we have $\rho_{2}=k_{1}((r / g)-\varepsilon) \rho_{1} / \alpha+g$ such that $\liminf _{t \longrightarrow+\infty} Y(t) \geq \rho_{2}$. Denote $\rho=\min \left\{\rho_{1}, \rho_{2}\right\}$; then, $\liminf _{t \longrightarrow+\infty} Y(t) \geq \rho$ and $\liminf _{t \longrightarrow+\infty} x_{2}(t) \geq \rho$. The proof of Theorem 4 is completed.

\section{Dynamics of the Model with Pulse Perturbation}

In this section, we extend model (1) by replacing the continuous removing of infected plants with a periodic pulse roguing control strategy, which is more realistic. Hence, we consider the following differential equation with pulse roguing strategy at fixed moments: 


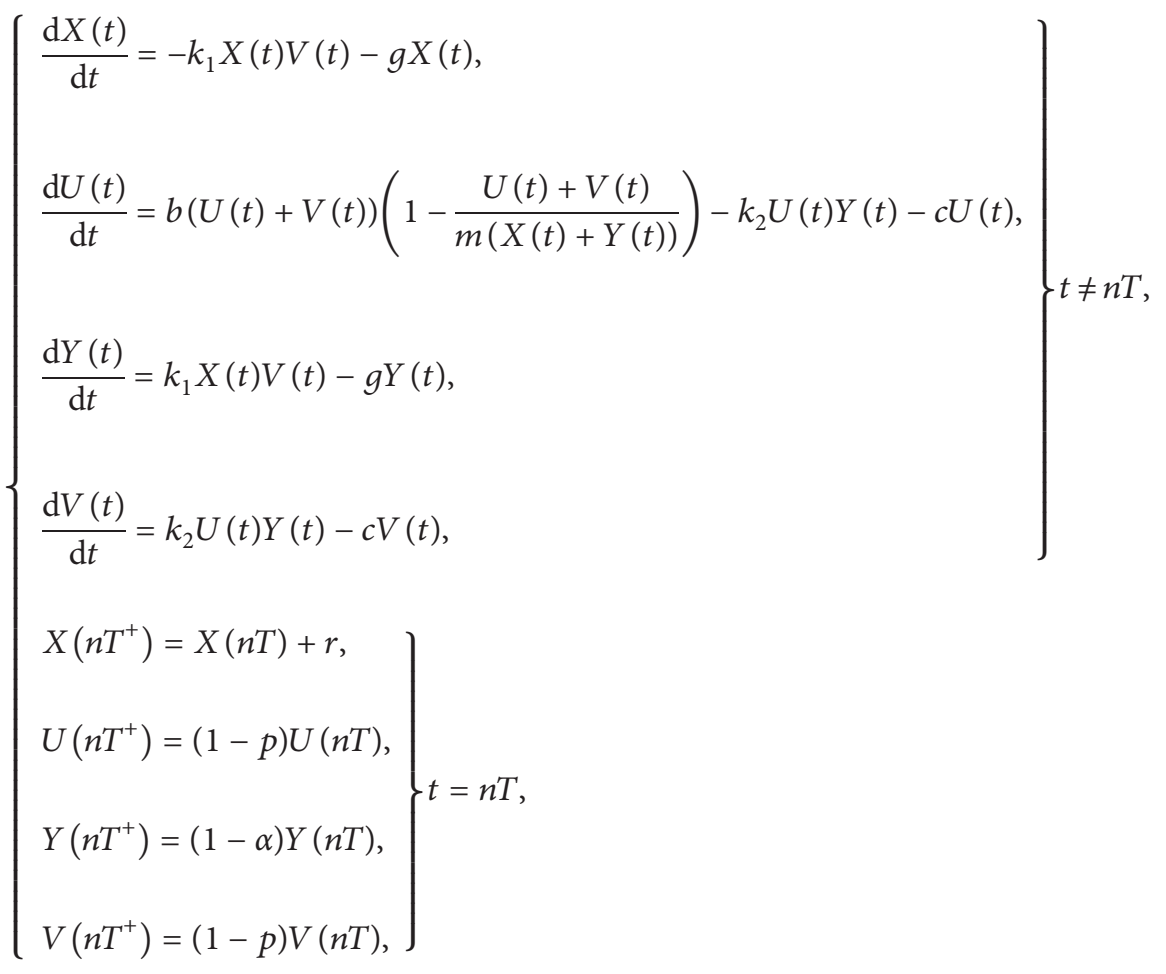

$$
X_{n+1}=X_{n} \exp (-g T)+r .
$$

where $T$ is a fixed positive constant and denotes the period of the impulsive effect, $r$ is the constant replanting parameter, and $n \in N$ which denotes the positive integer set. The parameter $\alpha$ denotes the proportion of the infected plants which is rouged at each pulse perturbation, and $p$ represents vectors removal rate due to the infected plants being rouged; it is likely that some of the uninfected vectors and infected vectors will be removed incidentally.

4.1. The Existence and Stability of the Disease-Free Periodic Solution. If $Y(t)=0, V(t)=0$, then model (25) can be reduced to the following subsystem:

$$
\left\{\begin{array}{l}
\frac{\mathrm{d} X(t)}{\mathrm{d} t}=-g X(t), \\
\frac{\mathrm{d} U(t)}{\mathrm{d} t}=b U(t)\left(1-\frac{U(t)}{m X(t)}\right)-c U(t),
\end{array}\right\} t \neq n T,
$$

From the first equation of (26), we can solve it in any impulsive interval $(n T,(n+1) T]$ and have

$$
X(t)=X\left(n T^{+}\right) \exp (-g(t-n T)), t \in(n T,(n+1) T] .
$$

Denote $X_{n}=X\left(n T^{+}\right)$; then,
There exists a steady state $X^{*}=r / 1-\exp (-g T)$, which indicates that the system

$$
\begin{cases}\frac{\mathrm{d} X(t)}{\mathrm{d} t}=-g X(t), & t \neq n T, \\ X\left(n T^{+}\right)=X(n T)+r, \quad t=n T \\ X\left(0^{+}\right)=X_{0}\end{cases}
$$

has a positive periodic solution $X^{*}(t)=X^{*} \exp (-g$ $(t-n T))$, which is globally asymptotically stable.

Lemma 2. System (29) has a positive periodic solution $X^{*}(t)$; for any solution of $(29)$, we have $X(t) \longrightarrow X^{*}(t)$ as $t \longrightarrow \infty$, where $X^{*}(t)=r \exp (-g(t-n T)) / 1-\exp (-g T), t \in(n T$, $(n+1) T]$.

Proof. Let $X(t)=X\left(n T^{+}\right) \exp (-g(t-n T))$ for all $t \in(n T$, $(n+1) T$ be any solution of $(29)$; then,

$$
X\left(n T^{+}\right)=X\left(0^{+}\right) \exp (-n g T)+\frac{r(1-\exp (-n g T))}{1-\exp (-g T)},
$$

which indicates that $X(t)=\left(X\left(0^{+}\right) \exp (-n g T)+r(1-\exp \right.$ $(-n g T)) / 1-\exp (-g T)) \exp (-g(t-n T)) \longrightarrow r \exp (-g(t \quad-$ $n T)) / 1-\exp (-g T)$ as $n \longrightarrow \infty$ and $t \in(n T,(n+1) T]$.

Substituting the above periodic solution into the second equation of (26), one yields 


$$
U(t)=\frac{m(g+b-c) X^{*} U\left(n T^{+}\right) \exp ((b-c)(t-n T))}{m(g+b-c) X^{*}+b U\left(n T^{+}\right)(\exp ((g+b-c)(t-n T))-1)}, \quad t \in(n T,(n+1) T] .
$$

Denote $U_{n}=U\left(n T^{+}\right)$; then,

$$
U_{n+1}=\frac{(1-p) m(g+b-c) \exp ((b-c) T) X^{*} U_{n}}{m(g+b-c) X^{*}+b U_{n}(\exp ((g+b-c) T)-1)} .
$$

Denote $R_{1}=(1-p) \exp ((b-c) T)$; then, we conclude that (32) always has a zero equilibrium provided that $R_{1}<1$ or $g+b-c=0$. When $R_{1}>1$ and $g+b-c \neq 0$, (32) has a stable positive equilibrium $U^{*}=m r(g+b-c)((1-p) \exp$ $((b-c) T)-1) / b(\exp ((g+b-c) T)-1)(1-\exp \quad(-g T))$.

$$
\begin{cases}\frac{\mathrm{d} U(t)}{\mathrm{d} t}=b U(t)\left(1-\frac{U(t)}{m X^{*}(t)}\right)-c U(t), & t \neq n T, \\ U\left(n T^{+}\right)=(1-p) U(n T), & t=n T, \\ U\left(0^{+}\right)=U_{0} & \end{cases}
$$

has a positive periodic solution

$$
U^{*}(t)=\frac{m(g+b-c) X^{*} U^{*} \exp ((b-c)(t-n T))}{m(g+b-c) X^{*}+b U^{*}(\exp ((g+b-c)(t-n T))-1)}, \quad t \in(n T,(n+1) T],
$$

which is globally asymptotically stable. Furthermore, we have the following main result.
Lemma 3. If $R_{1}>1$ and $g+b-c \neq 0$, then system (33) has $a$ positive periodic solution $U^{*}(t)$, and for any solution of (33) we have $U(t) \longrightarrow U^{*}(t)$ as $t \longrightarrow \infty$.

Proof. Denote $\bar{U}(t)=1 / U(t)$ and $\bar{U}(t)=1 / U^{*}(t)$; then,

$$
\begin{aligned}
\bar{U}(t)= & \bar{U}\left(n T^{+}\right) \exp (-(b-c)(t-n T)) \\
& +\frac{b(1-\exp (-g T))(\exp ((g+b-c)(t-n T))-1) \exp (-(b-c)(t-n T))}{m r(g+b-c)}, \\
\bar{U}^{*}(t)= & \bar{U}^{*} \exp (-(b-c)(t-n T)) \\
& +\frac{b(1-\exp (-g T))(\exp ((g+b-c)(t-n T))-1) \exp (-(b-c)(t-n T))}{m r(g+b-c)} .
\end{aligned}
$$

Thus, we have

$$
\begin{aligned}
\left|\bar{U}(t)-\bar{U}^{*}(t)\right| & =\left|\bar{U}\left(n T^{+}\right)-\bar{U}^{*}\right| \exp (-(b-c)(t-n T)) \\
& \leq\left|\bar{U}\left(n T^{+}\right)-\bar{U}^{*}\right| .
\end{aligned}
$$

Note that if $R_{1}>1$, then we have $\left|\bar{U}\left(n T^{+}\right)-\bar{U}^{*}\right|=\mid U$ $\left(n T^{+}\right)-U^{*} / U\left(n T^{+}\right) U^{*} \mid \longrightarrow 0$ as $t \longrightarrow \infty$, and consequently the result of Lemma 2 follows.

From Lemmas 1 and 2, we obtain the following results.

Lemma 4. System (26) has boundary periodic solution $\left(X^{*}(t), 0\right)$, which is globally stable if $R_{1} \leq 1$, and has a positive periodic solution $\left(X^{*}(t), U^{*}(t)\right)$, which is globally stable if $R_{1}>1$.

Therefore, system (25) has a plant-only periodic solution $\left(X^{*}(t), 0,0,0\right)$ and disease-free periodic solution $\left(X^{*}(t)\right.$, $\left.U^{*}(t), 0,0\right)$, and their stabilities have been addressed in the following.

Theorem 5. The plant-only periodic solution $\left(X^{*}(t), 0,0,0\right)$ of model (25) is globally asymptotically stable in the first quadrant if $R_{1}=(1-p) \exp ((b-c) T)<1$.

Proof. Firstly, we prove the local stability of the solution $\left(X^{*}(t), 0,0,0\right)$ of model (25). Denote $X(t)=x(t)+X^{*}(t)$, 
$Y(t)=y(t), U(t)=u(t), V(t)=v(t)$; the corresponding linear system of $(25)$ at $\left(X^{*}(t), 0,0,0\right)$ reads as

$$
\left\{\begin{array}{l}
\frac{\mathrm{d} x(t)}{\mathrm{d} t}=-k_{1} X^{*}(t) v(t)-g x(t), \\
\frac{\mathrm{d} u(t)}{\mathrm{d} t}=(b-c) u(t), \\
\frac{\mathrm{d} y(t)}{\mathrm{d} t}=k_{1} X^{*}(t) v(t)-g y(t), \\
\mathrm{d} t \\
x\left(n T^{+}\right)=x(n T)+r, \\
u\left(n T^{+}\right)=(1-p) u(n T), \\
y\left(n T^{+}\right)=(1-\alpha) y(n T), \\
v\left(n T^{+}\right)=(1-p) v(n T),
\end{array}\right\} t=n T,
$$

Thus, the fundamental solution matrix $\Phi(t)$ of (37) satisfies

$$
\frac{\mathrm{d} \Phi(t)}{\mathrm{d} t}=\left(\begin{array}{cccc}
-g & 0 & 0 & -k_{1} X^{*}(t) \\
0 & b-c & 0 & 0 \\
0 & 0 & -g & k_{1} X^{*}(t) \\
0 & 0 & 0 & -c
\end{array}\right) \Phi(t)
$$

and $\Phi(0)=I_{4 \times 4}$ is the identity matrix, which results in

$$
\Phi(t)=\left(\begin{array}{cccc}
e^{-g T} & 0 & 0 & \Delta_{14} \\
0 & e^{(b-c) T} & 0 & 0 \\
0 & 0 & e^{-g T} & \Delta_{34} \\
0 & 0 & 0 & e^{-c T}
\end{array}\right)
$$

There is no need to give the exact forms of $\Delta_{14}$ and $\Delta_{34}$ as they are not required in the analysis that follows. If all eigenvalues of

$$
M=\left(\begin{array}{cccc}
1 & 0 & 0 & 0 \\
0 & 1-p & 0 & 0 \\
0 & 0 & 1-\alpha & 0 \\
0 & 0 & 0 & 1-p
\end{array}\right) \Phi(T)
$$

are less than one, then the plant-only periodic solution $\left(X^{*}(t), 0,0,0\right)$ is locally stable. It is easy to see that

$$
\begin{aligned}
& \lambda_{1}=\exp (-g T)<1, \\
& \lambda_{2}=(1-p) \exp ((b-c) T), \\
& \lambda_{3}=(1-p) \exp (-g T)<1, \\
& \lambda_{4}=(1-p) \exp (-c T)<1,
\end{aligned}
$$

and then $\left|\lambda_{2}\right|<1$ if and only if $R_{1}<1$. According to Floquet's theory of impulsive differential equations, the plant-only periodic solution $\left(X^{*}(t), 0,0,0\right)$ is locally stable.

In the following, we prove the global attractivity of the periodic solution $\left(X^{*}(t), 0,0,0\right)$. It follows from $(25)$ that

$$
\left\{\begin{array}{l}
\frac{\mathrm{d} N(t)}{\mathrm{d} t}=-g N(t), \quad t \neq n T \\
N\left(n T^{+}\right) \leq N(n T)+r, \quad t=n T \\
N\left(0^{+}\right)=N_{0}
\end{array}\right.
$$

$$
\begin{cases}\frac{\mathrm{d} M(t)}{\mathrm{d} t}=b M(t)\left[1-\frac{M(t)}{m N(t)}\right]-c M(t), & t \neq n T, \\ M\left(n T^{+}\right)=(1-p) M(n T), & t=n T, \\ M\left(0^{+}\right)=M_{0} . & \end{cases}
$$




$$
\begin{aligned}
N(n T) & \leq N_{0} \exp (-n g T)+\frac{r(1-\exp (-n g T))}{1-\exp (-g T)} \\
& \longrightarrow \frac{r}{1-\exp (-g T)}, \quad \text { for } n \longrightarrow \infty
\end{aligned}
$$

Thus, $N(t)$ is uniformly bounded, and for $\varepsilon_{1}>0$ small enough, there exists a $t_{1}>0$ such that $X(t), Y(t) \leq L_{1}$, where $L_{1}=r / 1-\exp (-g T)+\varepsilon_{1}$. From (43), we get

$$
\begin{aligned}
M((n+1) T) & \leq \frac{(1-p) \exp ((b-c) T) M(n T)}{1+b(1-p)(\exp ((b-c) T)-1) M(n T) / m(b-c)\left(L_{1}+\varepsilon_{1}\right)} \\
& \leq(1-p) \exp ((b-c) T) M(n T) \\
& \leq((1-p) \exp ((b-c) T))^{n} M(0) \longrightarrow 0, \quad \text { for } n \longrightarrow \infty
\end{aligned}
$$

Thus, if $R_{1}<1$, then $M(t)$ is uniformly bounded, and for $\varepsilon_{2}>0$ small enough, there exists a $t_{2}>0$ such that $U(t), V(t) \leq \varepsilon_{2}$. Let $\varepsilon_{2} \longrightarrow 0$; then, $U(t) \longrightarrow 0, V(t) \longrightarrow 0$ as $\quad t \longrightarrow \infty$. From (25), $\mathrm{d} X(t) / \mathrm{d} t \leq-g T$, $X\left(n T^{+}\right)=X(n T)+r$. Consider the following impulsive differential equation:

$$
\begin{cases}\frac{\mathrm{d} Z_{1}(t)}{\mathrm{d} t}=-g Z_{1}(t), & t \neq n T, \\ Z_{1}\left(n T^{+}\right)=Z_{1}(n T)+r, \quad t=n T, \\ Z_{1}\left(0^{+}\right)=X\left(0^{+}\right)\end{cases}
$$

It follows from Lemma 1 and the comparison theorem on impulsive differential equations [38] that we have
$X(t) \leq Z_{1}(t)$ and $Z_{1}(t) \longrightarrow X^{*}(t)$ as $t \longrightarrow \infty$. Hence, for $\varepsilon_{3}>0$ small enough and all large $t$, we have

$$
X(t) \leq Z_{1}(t)<X^{*}(t)+\varepsilon_{3} .
$$

For simplification, we may assume that (47) holds for all $t \geq 0$. From (25), we get

$$
\begin{cases}\frac{\mathrm{d} Y(t)}{\mathrm{d} t} \leq k_{1}\left(X^{*}(t)+\varepsilon_{3}\right) \varepsilon_{2}-g Y(t), & t \neq n T, \\ Y\left(n T^{+}\right)=(1-\alpha) Y(n T), & t=n T, \\ Y\left(0^{+}\right)=Y_{0} . & \end{cases}
$$

Again, from the comparison theorem on impulsive differential equation, we get

$$
\begin{aligned}
Y((n+1) T) & \leq Y\left(n T^{+}\right) \exp (-g T)+\exp (-g T) \int_{n T}^{(n+1) T} k_{1}\left(X^{*}(t)+\varepsilon_{3}\right) \varepsilon_{2} \exp (g T) \mathrm{d} t \\
& =Y\left(n T^{+}\right) \exp (-g T)+k_{1} \varepsilon_{2} \int_{n T}^{(n+1) T}\left[\frac{r \exp (-g T)}{1-\exp (-g T)}+\varepsilon_{3}\right] \mathrm{d} t \\
& =Y\left(n T^{+}\right) \exp (-g T)+\frac{\left(\varepsilon_{3}+r \exp (-g T)\right) k_{1} \varepsilon_{2} T}{1-\exp (-g T)} \\
& =(1-\alpha) Y(n T) \exp (-g T)+\frac{\left(\varepsilon_{3}+r \exp (-g T)\right) k_{1} \varepsilon_{2} T}{1-\exp (-g T)} Y((n) T) \\
& \leq(1-\alpha)^{n} \exp (-n g T)+\frac{\left(\varepsilon_{3}+r \exp (-g T)\right) k_{1} \varepsilon_{2} T}{1-\exp (-g T)} \frac{1}{1-(1-\alpha) \exp (-g T)} .
\end{aligned}
$$

Let $\varepsilon_{2} \longrightarrow 0$; then, $Y(n T) \longrightarrow 0$ as $n \longrightarrow \infty$. Therefore, $Y(t) \longrightarrow 0$ as $t \longrightarrow \infty$.
Next, we show $X(t) \longrightarrow X^{*}(t)$ as $t \longrightarrow \infty$. It follows from $V(t)<\varepsilon_{2}$ that for $t>t_{2}$ we have 


$$
\frac{\mathrm{d} X(t)}{\mathrm{d} t} \geq-k_{1} X(t) \varepsilon_{2}-g X(t)
$$

then, we have

$$
\begin{cases}\frac{\mathrm{d} Z_{2}(t)}{\mathrm{d} t}=-\left(k_{1} \varepsilon_{2}+g\right) Z_{2}(t), & t \neq n T, \\ Z_{2}\left(n T^{+}\right)=Z_{2}(n T)+r, & t=n T, \\ Z_{2}\left(0^{+}\right)=X\left(0^{+}\right) . & \end{cases}
$$

Note that the above system has a globally stable periodic solution $\quad Z_{2}^{*}(t)=r \exp \left(-\left(k_{1} \varepsilon_{2}+g\right)(t-n T)\right) / 1-\exp$ $\left(\left(-\left(k_{1} \varepsilon_{2}+g\right) T\right)\right.$ with $Z_{2}^{*}\left(n T^{+}\right)=r / 1-\exp \left(\left(-\left(k_{1} \varepsilon_{2}+g\right) T\right)\right.$. From the comparison theorem, we get $Z_{2}(t) \leq X(t)$ $\leq Z_{1}(t), Z_{2}(t) \longrightarrow Z_{2}^{*}(t)$ and $Z_{1}(t) \longrightarrow X^{*}(t)$ as $t \longrightarrow \infty$. Therefore, there exists a $t_{3}$ such that $t_{3}>t_{2}$, and for $t>t_{3}$ we have

$$
Z_{2}^{*}(t)-\varepsilon_{3}<X(t)<X^{*}(t)+\varepsilon_{3},
$$

with $\varepsilon_{3}>0$ small enough.

Let $\varepsilon_{2} \longrightarrow 0$, so that $Z_{2}(t) \longrightarrow X^{*}(t)$. Then, (52) becomes

$$
X^{*}(t)-\varepsilon_{3}<X(t)<X^{*}(t)+\varepsilon_{3} .
$$

Hence, $X(t) \longrightarrow X^{*}(t)$ as $t \longrightarrow \infty$. This completes the proof.

Next, to investigate the stability results of disease-free periodic solution $\left(X^{*}(t), U^{*}(t), 0,0\right)$ of model (25), we first calculate the basic reproduction number for the impulsive system (25) by using the next infection operator for the piecewise continuous periodic system proposed in $[39,40]$. Denote $\quad X(t)=x(t)+X^{*}(t), Y(t)=y(t), U(t)=u(t)$ $+U^{*}(t), V(t)=v(t)$. The corresponding linear system (25) at $\left(X^{*}(t), U^{*}(t), 0,0\right)$ reads as

$$
\begin{cases}W \prime(t)=G(t) W(t), & t \neq n T, \\ W\left(n T^{+}\right)=H W(n T), & t=n T .\end{cases}
$$

In this system, we have $W(t)=(x(t), u(t), y(t), v(t))^{T}$,

$$
\begin{aligned}
G(t) & =\left(\begin{array}{cc}
G_{1}(t) & G_{2}(t) \\
0 & F(t)-V_{r}(t)
\end{array}\right), \\
H & =\left(\begin{array}{cc}
H_{1} & 0 \\
0 & H_{2}
\end{array}\right),
\end{aligned}
$$

with

$$
\begin{aligned}
F(t) & =\left(\begin{array}{cc}
0 & k_{1} X^{*}(t) \\
k_{2} U^{*}(t) & 0
\end{array}\right), \\
V_{r}(t) & =\left(\begin{array}{ll}
g & 0 \\
0 & c
\end{array}\right), 0=\left(\begin{array}{ll}
0 & 0 \\
0 & 0
\end{array}\right), \\
G_{1}(t) & =\left(\begin{array}{cc}
-g & 0 \\
\frac{b U^{* 2}(t)}{m X^{* 2}(t)} b-c-\frac{2 b U^{*}(t)}{m X^{*}(t)}
\end{array}\right), \\
G_{2}(t) & =\left(\begin{array}{cc}
b U^{*}(t) \\
\frac{b X^{* 2}(t)}{m}-k_{2} U^{*}(t) & b-\frac{2 b U^{*}(t)}{m X^{*}(t)}
\end{array}\right) \\
H_{1} & =\left(\begin{array}{cc}
1 & 0 \\
0 & 1-p
\end{array}\right), H_{2}=\left(\begin{array}{cc}
1-\alpha & 0 \\
0 & 1-p
\end{array}\right) .
\end{aligned}
$$

Let $\Phi_{G}(t)=\left(\Phi_{i j}\right), 1 \leq i, j \leq 2$, be the fundamental solution matrix of (54). Consequently, we have $\Phi_{G}^{\prime}(t)=G(t) \Phi_{G}(t)$ with appropriate initial value $\Phi_{G}(0)=I_{4}$. Further computation implies that

$$
\Phi_{G}(t)=\left(\begin{array}{cc}
\exp \left(G_{1}(t)\right) & \Phi_{12}(t) \\
0 & \Phi_{F(t)-V_{r}(t)}(t)
\end{array}\right)
$$

then, we obtain

$$
H \Phi_{G}(T)=\left(\begin{array}{cc}
H_{1} \exp \left(G_{1}(T)\right) & H_{1} \Phi_{12}(T) \\
0 & H_{2} \Phi_{F(t)-V_{r}(t)}(T)
\end{array}\right) .
$$

By $[39,40]$, the basic reproduction number for system (54) is given as follows:

$$
R_{2}=\rho\left(H_{2} \Phi_{F(t)-V_{r}(t)}(T)\right) .
$$

Then, based on Floquet theory, we have the following conclusion.

Theorem 6. The disease-free periodic solution $\left(X^{*}(t), U^{*}(t), 0,0\right)$ of model $(25)$ is globally asymptotically stable in the first quadrant if $R_{1}=\left(1-p_{1}\right) \exp ((b-c) T)>1$ and $R_{2}<1$.

Proof. To prove the global stability of $\left(X^{*}(t), U^{*}(t), 0,0\right)$, we only need to show the global attractivity. Suppose $R_{1}>1$; then, from the boundedness of (25), there exists a $t_{1}>0$ such that $X(t), Y(t) \leq L_{1}$ and $U(t), V(t) \leq L_{2}$ with $t \geq t_{1}$ for every solution $(X(t), Y(t), U(t), V(t))$ of $(4.1)$, where 


$$
\begin{aligned}
& L_{1}=\frac{r}{1-\exp (-g T)}+\varepsilon_{1}, \\
& L_{2}=\frac{b(1-\exp (-(b-c) T))}{m L_{1}(b-c)(1-\exp (-(b-c) T))}+\varepsilon .
\end{aligned}
$$

It follows from (25) that

$$
\begin{cases}\frac{\mathrm{d} X(t)}{\mathrm{d} t} \leq-g X(t), & t \neq n T, \\ X\left(n T^{+}\right)=X(n T)+r, \quad t=n T \\ X\left(0^{+}\right)=X_{0}\end{cases}
$$

Consider the following system:

$$
\begin{cases}\frac{\mathrm{d} Z_{3}(t)}{\mathrm{d} t}=-g Z_{3}(t), & t \neq n T, \\ Z_{3}\left(n T^{+}\right)=Z_{3}(n T)+r, & t=n T, \\ Z_{3}\left(0^{+}\right)=X\left(0^{+}\right) . & \end{cases}
$$

From Lemma 1 and the comparison theorem on impulsive differential equations [38], we have $X(t) \leq Z_{3}(t)$ and
$Z_{3}(t) \longrightarrow X^{*}(t)$ as $t \longrightarrow \infty$. Hence, for $\varepsilon_{3}>0$ small enough and all large $t$, we have

$$
X(t) \leq Z_{3}(t)<X^{*}(t)+\varepsilon_{3} .
$$

From (25), we get

$$
\begin{cases}\frac{\mathrm{d} Y(t)}{\mathrm{d} t}=k_{1} X(t) V(t)-g Y(t) \doteq f_{1}(Y(t), V(t)), & t \neq n T, \\ \frac{\mathrm{d} V(t)}{\mathrm{d} t}=k_{2} U(t) Y(t)-c V(t) \doteq f_{2}(Y(t), V(t)), & t \neq n T, \\ Y\left(n T^{+}\right)=(1-\alpha) Y(n T), & t=n T, \\ V\left(n T^{+}\right)=(1-p) V(n T), & t=n T, \\ Y\left(0^{+}\right)=Y_{0} & \\ V\left(0^{+}\right)=V_{0} & \end{cases}
$$

Consider the following system:

$$
\begin{cases}\frac{\mathrm{d} Q_{1}(t)}{\mathrm{d} t}=k_{1}\left(X^{*}(t)+\varepsilon_{3}\right) Q_{2}(t)-g Q_{1}(t) \dot{=} g_{1}\left(Q_{1}(t), Q_{2}(t)\right), & t \neq n T, \\ \frac{\mathrm{d} Q_{2}(t)}{\mathrm{d} t}=k_{2} M^{*}(t) Q_{1}(t)-c Q_{2}(t) \dot{=} g_{2}\left(Q_{1}(t), Q_{2}(t)\right), & t \neq n T, \\ Q_{1}\left(n T^{+}\right)=(1-\alpha) Q_{1}(n T), & t=n T, \\ Q_{1}\left(n T^{+}\right)=(1-p) Q_{2}(n T), & t=n T, \\ Q_{1}\left(0^{+}\right)=Y_{0}, & \\ Q_{2}\left(0^{+}\right)=V_{0}, & \end{cases}
$$

which is equivalent to

$$
\begin{cases}\frac{\mathrm{d} Q(t)}{\mathrm{d} t}=\left(F(t)-V_{r}(t)\right) Q(t)+k_{1} \varepsilon_{3} I Q(t), & t \neq n T, \\ Q\left(n T^{+}\right)=H_{2} Q(n T), & t=n T,\end{cases}
$$

where $Q(t)=\left(Q_{1}(t), Q_{2}(t)\right)^{T}$ and $I$ is identity matrix. By $[39,40]$, there exists a positive $T$-periodic function $\nu(t)$, such that $Q(t)=\exp \left(\mu_{1} t\right) \nu(t)$ is a solution of system (68), where $\mu_{1}=1 / T \ln \rho\left(H_{2} \Phi_{\left(F-V_{r}+k_{1} \varepsilon_{3} I\right)}(T)\right)$. When $R_{2}<1$, we have
$\rho\left(H_{2} \Phi_{\left(F-V_{r}\right)}(T)\right)<1$. Due to the continuity of $\rho\left(H_{2} \Phi_{\left(F-V_{r}+k_{1} \varepsilon_{3} I\right)}(T)\right)$, we can choose sufficient small $\varepsilon_{3}$ such that $\rho\left(H_{2} \Phi_{\left(F-V_{r}+k_{1} \varepsilon_{3} I\right)}(T)\right)<1$. Hence, we obtain $\mu_{1}<0$, and $Q(t) \longrightarrow 0$ as $t \longrightarrow \infty$. For any nonnegative initial value $\left(Q_{1}\left(t_{2}\right), Q_{2} V\left(t_{2}\right)\right)^{T}$ of system (68), there exists an $\eta$ large enough such that

$$
\left(Q_{1}\left(t_{2}\right), Q_{2}\left(t_{2}\right)\right)^{T} \leq \eta\left(\nu_{1}(0), \nu_{2}(0)\right)^{T} .
$$

Since $\quad \partial g_{1} / \partial Q_{2}(t) \geq 0, \quad \partial g_{2} / \partial Q_{1}(t) \geq 0, \quad$ and $f_{i} \leq g_{i}, i=1,2$, system (68) is a quasimonotone increasing system [41]; by the comparison theorem [38], we obtain 


$$
\begin{array}{r}
(Y(t), V(t)) \leq \eta \exp \left(\mu_{1}\left(t-t_{2}\right)\right)\left(\nu_{1}\left(t-t_{2}\right), \nu_{2}\left(t-t_{2}\right)\right)^{T}, \\
t \geq t_{2} .
\end{array}
$$

Hence, we have $Y(t) \longrightarrow 0, V(t) \longrightarrow 0$ as $t \longrightarrow \infty$.

Next, we shall prove that if $\lim _{t \rightarrow \infty} Y(t) t=0$ and $\lim _{t \longrightarrow \infty} V(t)=0$, then $X(t) \longrightarrow X^{*}(t) \quad$ and $U(t) \longrightarrow U^{*}(t)$ as $t \longrightarrow \infty$. First, we show $U(t) \longrightarrow U^{*}(t)$ as $t \longrightarrow \infty$, since $M(t)=(U(t)+V(t)) \longrightarrow M^{*}(t)$, when $\lim _{t \rightarrow \infty} V(t)=0, U(t) \longrightarrow M^{*}(t)=U^{*}(t)$. It follows from $\lim _{t \rightarrow \infty} V(t)=0$ that, for $\varepsilon_{3}>0$ small enough, there exists a $t_{3}$ such that $t_{3} \geq t_{2}$ and $0<V(t)<\varepsilon_{3}$ for $t \geq t_{3}$. Thus, for $t \geq t_{3}$, we have

$$
\left(-k_{1} \varepsilon_{3}-g\right) X(t) \leq \frac{\mathrm{d} X(t)}{\mathrm{d} t} \leq-g X(t),
$$

from which the following system is obtained:

$$
\begin{cases}\frac{\mathrm{d} Z_{4}(t)}{d t}=\left(-k_{1} \varepsilon_{3}-g\right) Z_{4}(t), & t \neq n T, \\ Z_{4}\left(n T^{+}\right)=Z_{4}(n T)+r, & t=n T, \\ Z_{4}\left(0^{+}\right)=X\left(0^{+}\right) . & \end{cases}
$$

It follows from Lemma 1 that system (72) has a globally stable positive periodic solution $Z_{4}^{*}(t)$ for $t \in(n T$, $(n+1) T] ; \quad$ here, $\quad Z_{4}^{*}(t)=r \exp \left(\left(-k_{1} \varepsilon_{3}-g\right)(t-n T)\right) / 1-$ $\exp \left(\left(k_{1} \varepsilon_{3}-g\right) T\right)$ with $Z_{4}^{*}\left(n T^{+}\right)=r / 1-\exp \left(\left(k_{1} \varepsilon_{3}-g\right) T\right)$. By the comparison theorem, we obtain $Z_{4}(t) \leq X(t) \leq Z_{3}(t)$, $Z_{4}(t) \longrightarrow Z_{4}^{*}(t)$, and $Z_{3}(t) \longrightarrow X^{*}(t)$ as $t \longrightarrow \infty$. Therefore, there is a $t_{4}$ for $\varepsilon_{4}>0$ small enough such that $t_{4} \geq t_{3}$; for $t \geq t_{4}$, we have

$$
Z_{4}^{*}(t)-\varepsilon_{4} \leq X(t) \leq X^{*}(t)+\varepsilon_{4} .
$$

Let $\varepsilon_{3} \longrightarrow 0$ in $(71)$, so that $Z_{2}(t) \longrightarrow X^{*}(t)$. Then,

$$
X^{*}(t)-\varepsilon_{4} \leq X(t) \leq X^{*}(t)+\varepsilon_{4} .
$$

Hence, $X(t) \longrightarrow X^{*}(t)$ as $t \longrightarrow \infty$. We have proved the global attractivity of the disease-free periodic solution $\left(X^{*}(t), U^{*}(t), 0,0\right)$ of model (25). This completes the proof.

4.2. Permanence. The persistence of the system indicates that plant (susceptible and infected) and vectors (susceptible and infected) can coexist. If our goal is to eliminate infected plants and vectors, then the persistence suggests that control strategies fail to achieve it. Meanwhile, the permanent condition obtained from analyzing the system can provide scientific support for us to identify the key factors that result in failure and the effectiveness of control strategies, and then guide us to establish a good control program.

Theorem 7. System (25) is uniformly persistent if $R_{2}>1$.

Proof. It follows from $R_{2}>1$ that $\rho\left(H_{2} \Phi_{\left(F-V_{r}\right)(T)}\right)>1$, which indicates that there exists $\bar{\varepsilon}>0$ such that

$$
\rho\left(H_{2} \Phi_{\left(F-V_{r}-\bar{\varepsilon} M_{1}\right)}(T)\right)>1,
$$

where

$$
M_{1}=\left(\begin{array}{cc}
0 & k_{1} \\
k_{2} & 0
\end{array}\right) .
$$

Let $(X(t), U(t), Y(t), V(t))$ be any solution of (25). First, we claim that we can find a positive constant $\eta_{2}$ such that

$$
\begin{aligned}
& \lim _{t \longrightarrow \infty} \sup Y(t) \geq \eta_{2}, \\
& \lim _{t \longrightarrow \infty} \sup V(t) \geq \eta_{2} .
\end{aligned}
$$

Otherwise, there exists $t_{1}$ such that $Y(t)<\eta_{2}$ and $V(t)<\eta_{2}$ for all $t \geq t_{1}$. Based on system (25), we have

$$
\begin{cases}\frac{\mathrm{d} X(t)}{\mathrm{d} t}=-k_{1} V(t) X(t)-g X(t) \doteq f_{3}(X(t), U(t)), & t \neq n T, \\ \frac{\mathrm{d} U(t)}{\mathrm{d} t}=b U(t)\left(1-\frac{U(t)+\eta_{2}}{m X(t)}\right)-k_{2} \eta_{2} U(t)-c U(t) \doteq f_{4}(X(t), U(t)), & t \neq n T, \\ X\left(n T^{+}\right)=X(n T)+r, & t=n T, \\ U\left(n T^{+}\right)=(1-p) U(n T), & t=n T,\end{cases}
$$

and we take the auxiliary system 


$$
\begin{cases}\frac{\mathrm{d} \widetilde{X}(t)}{\mathrm{d} t}=-k_{1} \eta_{2} \widetilde{X}(t)-g \widetilde{X}(t) \doteq g_{3}(\widetilde{X}, \widetilde{U}(t)), & t \neq n T, \\ \frac{\mathrm{d} \widetilde{U}(t)}{\mathrm{d} t}=b \widetilde{U}(t)\left(1-\frac{\tilde{U}(t)+\eta}{m \widetilde{X}(t)}\right)-k_{2} \eta \widetilde{U}(t)-c \widetilde{U}(t) \dot{\doteq} g_{4}(\widetilde{X}(t), \widetilde{U}(t)), & t \neq n T, \\ \widetilde{X}\left(n T^{+}\right)=\widetilde{X}(n T)+r, & t=n T, \\ \widetilde{U}\left(n T^{+}\right)=(1-p) \widetilde{U}(n T), & t=n T .\end{cases}
$$

Since $\partial f_{3} / \partial U(t)=0, \partial f_{4} / \partial X(t) \geq 0$, and $f_{i} \geq g_{i}, i=3,4$, system (78) is a quasimonotone increasing system [41]. By the comparison theorem [38], we have $X(t) \geq \widetilde{X}(t)$, $U(t) \geq \widetilde{U}(t)$ and $\tilde{X}(t) \longrightarrow X^{*}(t), \widetilde{U}(t) \longrightarrow U^{*}(t)$ as $t \longrightarrow \infty$ and $\eta_{2} \longrightarrow 0$. Therefore, for the above $\bar{\varepsilon}>0$, there exist $t_{2} \geq t_{1}$ and $\tilde{\eta}>0$; for $\eta_{2}<\tilde{\eta}$, we have

$$
\begin{aligned}
& X(t) \geq \tilde{X}(t) \geq X^{*}(t)-\bar{\varepsilon} \\
& U(t) \geq \tilde{U}(t) \geq U^{*}(t)-\bar{\varepsilon} .
\end{aligned}
$$

Again, from system (25), we have

$$
\begin{cases}\frac{\mathrm{d} Y(t)}{\mathrm{d} t} \geq k_{1}\left(X^{*}(t)-\bar{\varepsilon}\right) V(t)-g Y(t), & t \neq n T, \\ \frac{\mathrm{d} V(t)}{\mathrm{d} t} \geq k_{2}\left(U^{*}(t)-\bar{\varepsilon}\right) Y(t)-c V(t), & t \neq n T, \\ Y\left(n T^{+}\right)=(1-\alpha) Y(n T), & t=n T, \\ V\left(n T^{+}\right)=(1-p) V(n T), & t=n T .\end{cases}
$$

Further, we consider an auxiliary system

$$
\begin{cases}\frac{\mathrm{d} \widetilde{Y}(t)}{\mathrm{d} t}=k_{1} X^{*}(t) \tilde{V}(t)-g \widetilde{Y}(t)-\bar{\varepsilon} \widetilde{V}(t), & t \neq n T, \\ \frac{\mathrm{d} \widetilde{V}(t)}{\mathrm{d} t}=k_{2} U^{*}(t) \widetilde{Y}(t)-c \widetilde{V}(t) \bar{\varepsilon} \widetilde{Y}(t), & t \neq n T, \\ \widetilde{Y}\left(n T^{+}\right)=(1-\alpha) \widetilde{Y}(n T), & t=n T, \\ \widetilde{V}\left(n T^{+}\right)=(1-p) \widetilde{V}(n T), & t=n T,\end{cases}
$$

which can be rewritten as

$$
\left\{\begin{array}{l}
\left(\begin{array}{l}
\frac{\mathrm{d} \tilde{Y}(t)}{\mathrm{d} t} \\
\frac{\mathrm{d} \widetilde{V}(t)}{\mathrm{d} t}
\end{array}\right)=\left(\left(F(t)-V_{r}(t)\right)-\bar{\varepsilon} M_{1}\right)\left(\begin{array}{c}
\tilde{Y}(t) \\
\tilde{V}(t)
\end{array}\right), \quad t \neq n T, \\
\left(\begin{array}{c}
\tilde{Y}\left(n T^{+}\right) \\
\tilde{V}\left(n T^{+}\right)
\end{array}\right)=H_{2}\left(\begin{array}{c}
\tilde{Y}(n T) \\
\tilde{V}(n T)
\end{array}\right),
\end{array}\right.
$$

where $F(t), V_{r}(t)$, and $H_{2}$ can be seen in (56) and (58). It follows from the results shown in $[39,40]$ that there exists a positive T-periodic function $W(t)=\left(W_{1}(t), W_{2}(t)\right)$ such that $v_{2}(t)=\exp \left(\mu_{2} t\right) W(t)$ is a solution of $(83)$, where $\mu_{2}=$ $1 / T \ln \rho\left(H_{2} \Phi_{\left(F-V_{r}-\bar{\varepsilon} M_{1}\right)}(T)\right)$ and $\nu_{2}(t)=(\widetilde{Y}(t), \widetilde{V}(t))$. From (75), $\mu_{2}>0$. Choose $t_{3} \geq t_{2}$ and $\eta_{2}>0$ such that $W\left(t_{2}\right) \geq \eta_{2} \nu_{2}(0)$; we obtain

$$
W(t) \geq \eta_{2} \exp \left(\mu_{2}\left(t-t_{2}\right)\right) v_{2}\left(t-t_{2}\right), \quad t \geq t_{3} .
$$

By the comparison theorem [38], we obtain that, for all $t \geq t_{3}$,

$$
\begin{aligned}
& Y(t) \geq \tilde{Y}(t) \geq \eta_{2} \exp \left(\mu_{2}\left(t-t_{2}\right)\right) v_{2}\left(t-t_{2}\right), \\
& V(t) \geq \widetilde{V}(t) \geq \eta_{2} \exp \left(\mu_{2}\left(t-t_{2}\right)\right) v_{2}\left(t-t_{2}\right)
\end{aligned}
$$

As a result, we obtain $W(t) \longrightarrow \infty$ as $t \longrightarrow \infty$. Consequently, $(\tilde{Y}(t), \tilde{V}(t)) \longrightarrow+\infty$ as $t \longrightarrow \infty$, which conflicts with $\tilde{Y}(t)<\eta_{2}$ and $\left.\tilde{V}(t)\right)<\eta_{2}$. Thus,

$$
\begin{aligned}
& \lim _{t \longrightarrow \infty} \sup Y(t) \geq \eta_{2}, \\
& \lim _{t \rightarrow \infty} \sup V(t) \geq \eta_{2} .
\end{aligned}
$$

Furthermore, we have the following two possibilities. 
(i) $Y(t) \geq \eta_{2}$ and $V(t) \geq \eta_{2}$ for all large $t$.

(ii) $Y(t)$ and $V(t)$ oscillations about $\eta_{2}$ for all large $t$.

If case (i) holds, then the result is obtained. Next, we consider case (ii). Because $\lim _{t \longrightarrow \infty} \sup Y(t) \geq \eta_{2}$ and $\lim _{t \rightarrow \infty} \sup V(t) \geq \eta_{2}$, we can choose a $t_{1} \in\left(n_{1} T,\left(n_{1}+1\right) T\right]$ such that $Y\left(t_{1}\right) \geq \eta_{2}$ and $V\left(t_{1}\right) \geq \eta_{2}$. The above discussion implies that there exists $t_{2} \in\left(n_{2} T,\left(n_{2}+1\right) T\right]$, such that $Y\left(t_{2}\right) \geq \eta_{2}$ and $V\left(t_{2}\right) \geq \eta_{2}$, where $n_{2}-n_{1} \geq 0$ is finite. Then, we will consider the solution of system (25) in the interval $\left[t_{1}, t_{2}\right]$ :

$$
\frac{\mathrm{d} Y(t)}{\mathrm{d} t}=k_{1} X(t) V(t)-g Y(t) \geq-g Y(t),
$$

which results in the following relations:

$$
\begin{aligned}
Y(t) & \geq Y\left(t_{1}\right) \exp \left(-g\left(t-t_{1}\right)\right) \geq \eta_{2} \exp \left(-g\left(t_{2}-t_{1}\right)\right) \\
& \geq \eta_{2} \exp \left(-g\left(n_{2}-n_{1}\right) T\right) .
\end{aligned}
$$

Similarly, it follows from

$$
\frac{\mathrm{d} V(t)}{\mathrm{d} t}=k_{2} U(t) Y(t)-c V(t) \geq-c V(t)
$$

that we have

$$
\begin{aligned}
V(t) & \geq V\left(t_{1}\right) \exp \left(-c\left(t-t_{1}\right)\right) \geq \eta_{2} \exp \left(-c\left(t_{2}-t_{1}\right)\right) \\
& \geq \eta_{2} \exp \left(-c\left(n_{2}-n_{1}\right) T\right) .
\end{aligned}
$$

Let $\quad \sigma_{1}=\min \left\{\eta_{2} \exp \left(-g\left(n_{2}-n_{1}\right) T\right), \eta_{2} \exp \left(-c\left(n_{2}-\right.\right.\right.$ $\left.\left.\left.n_{1}\right) T\right)\right\}$; then, $\sigma_{1}>0$ cannot be infinitely small and $n_{2}-n_{1} \geq 0$ is finite. We get $Y(t) \geq \sigma_{1}>0$ and $V(t) \geq \sigma_{1}>0$.

For $t>t_{2}$, we take the same steps and get another positive $\sigma_{2}$. As a consequence of this, we obtain the sequence $\sigma_{i}$, where $\quad \sigma_{i}=\min \left\{\eta_{2} \exp \left(-g\left(n_{i+1}-n_{i}\right) T\right), \eta_{2} \exp \left(-c\left(n_{2}-\right.\right.\right.$ $\left.\left.\left.n_{1}\right) T\right)\right\}$, for $i=1,2, \ldots$, is noninfinitesimal when $n_{i+1}-n_{i} \geq 0$ is finite. In this case, the solution of system (25) satisfies $Y(t) \geq \sigma_{i}>0$ and $V(t) \geq \sigma_{i}>0$ for all $\left[t_{i}, t_{i+1}\right]$, where $t_{i} \in\left(n_{i} T, n_{i+1} T\right]$. Let $\sigma=\min \left\{\sigma_{i}, i=1,2, \ldots,\right\}$; we obtain $Y(t) \geq \sigma>0$ and $V(t) \geq \sigma>0$. This completes the proof.

\section{Optimum Times of Roguing Control}

Note that, in reality, replanting the susceptible and removing the infected plants are usually not at the same time, and the infected plants could be removed several times within each replanting period. The key question is whether there exist optimal times of removing the infected plants within each replanting period such that the threshold value reaches its minimum. To address this question, we extend model (25) by taking the multiple pulse controls as follows:

$$
\left\{\begin{aligned}
\frac{\mathrm{d} X(t)}{\mathrm{d} t} & =-k_{1} X(t) V(t)-g X(t), \\
\frac{\mathrm{d} U(t)}{\mathrm{d} t} & =b(U(t)+V(t))\left(1-\frac{U(t)+V(t)}{m(X(t)+Y(t))}\right)-k_{2} U(t) Y(t)-c U(t), \\
\frac{\mathrm{d} Y(t)}{\mathrm{d} t} & =k_{1} X(t) V(t)-g Y(t), \\
\frac{\mathrm{d} V(t)}{\mathrm{d} t} & =k_{2} U(t) Y(t)-c V(t), \\
X\left(t^{+}\right) & =X(t)+r, \\
U\left(t^{+}\right) & =U(t), \\
Y\left(t^{+}\right) & =Y(t), \\
V\left(t^{+}\right) & =V(t), \\
X\left(t^{+}\right) & =X(t), \\
U\left(t^{+}\right) & =(1-p) U(t), \\
Y\left(t^{+}\right) & =(1-\alpha) Y(t), \\
V\left(t^{+}\right) & =(1-p) V(t),
\end{aligned}\right\}, \quad t=\left\{\tau_{n}, t_{n}^{i}\right\},
$$


where $\tau_{n}(n=0,1,2, \ldots$,$) and t_{n}^{i}(i=1,2, \ldots, k)$ are impulsive point series at which the replanting and removing actions have been taken, respectively. Further, we assume $\tau=\tau_{n+1}-\tau_{n}$ for all $n=0,1,2, \ldots$, where $\tau$ is the period of replanting. For this case, model (91) is called a period system with period $\tau$ if there exists a positive integer $k$ such that $t_{n+1}^{k}-t_{n}^{k}=\tau$, where $t_{n}^{i} \in\left[\tau_{n}, \tau_{n+1}\right]$ for all $i=1,2, \ldots, k$. This implies that, in each period $\tau$, the infected plants have been removed $k$ times. Denote $\Delta_{i}=t_{n}^{i+1}-t_{n}^{i}, i=0,1,2, \ldots, k$, where $\Delta_{0}=\tau_{n}, \Delta_{k+1}=\tau_{n+1}-t_{n}^{k}$. Firstly, the basic properties of subsystem

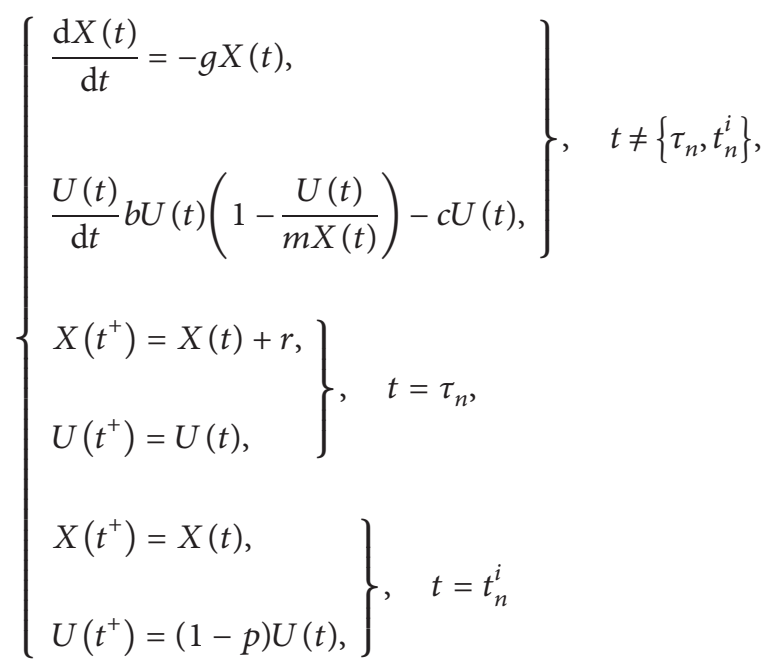

play a key role in analyzing the disease control.

In any given time interval $\left(\tau_{n}, \tau_{n+1}\right]$, we integrate the first equation of system (92) and have $X(t)=X\left(\tau_{n}^{+}\right) \exp \left(-g\left(t-\tau_{n}\right)\right)$. At time $\tau_{n+1}$, one replanting strategy is applied, which results in

$$
X\left(\tau_{n+1}^{+}\right)=X\left(\tau_{n}^{+}\right) \exp (-g \tau)+r .
$$

Denote $X_{\tau_{n}}=X\left(\tau_{n}^{+}\right)$; then, we have the following difference equation: $X_{\tau_{\text {ut }}}=X_{\tau_{n}} \exp (-g \tau)+r$, which has a unique steady state $\frac{\tau_{u_{* * 1}}}{X^{*}}=r / 1^{n}-\exp (-g \tau)$. Therefore, from Lemma 1, the subsystem

$$
\left\{\begin{array}{l}
\frac{\mathrm{d} X(t)}{\mathrm{d} t}=-g X(t), \quad t \neq \tau_{n}, \\
X\left(t^{+}\right)=X(t)+r, \quad t=\tau_{n}, \\
X\left(0^{+}\right)=X_{0}
\end{array}\right.
$$

has a positive periodic solution $\bar{X}^{*}(t)=\bar{X}^{*} \exp \left(-g\left(t-\tau_{n}\right)\right)$ which is globally asymptotically stable.

Further, substituting the above solution into the second equation of (92) and integrating the second equation of system (92) from $\tau_{n}$ to $t_{n}^{1}$ yields

$$
U(t)=\frac{U\left(\tau_{n}^{+}\right) \exp \left((b-c)\left(t-\tau_{n}\right)\right)}{1+b U\left(\tau_{n}^{+}\right) / m(g+b-c) \bar{X}^{*}\left(\exp \left((g+b-c)\left(t-\tau_{n}\right)\right)-1\right)}, \quad t \in\left(\tau_{n}, t_{n}^{1}\right]
$$

At time $t_{n}^{1}$, one removing strategy is applied, which results in

$$
U\left(t_{n}^{1+}\right)=\frac{(1-p) U\left(\tau_{n}^{+}\right) \exp \left((b-c) \Delta_{1}\right)}{1+b U\left(\tau_{n}^{+}\right) / m(g+b-c) \bar{X}^{*}\left(\exp \left((g+b-c) \Delta_{1}\right)-1\right)} .
$$

Again, integrating the second equation of system (92) from $t_{n}^{1}$ to $t_{n}^{2}$ yields

$$
U(t)=\frac{U\left(t_{n}^{1+}\right) \exp \left((b-c)\left(t-t_{n}^{1}\right)\right)}{1+b U\left(t_{n}^{1+}\right) / m(g+b-c) \bar{X}^{*}\left(\exp \left((g+b-c)\left(t-t_{n}^{1}\right)\right)-1\right)}, \quad t \in\left(t_{n}^{1}, t_{n}^{2}\right]
$$


Further, at time $t_{n}^{2}$, one removing tactics is applied and

$$
U\left(t_{n}^{2+}\right)=\frac{(1-p)^{2} U\left(\tau_{n}^{+}\right) \exp \left((b-c)\left(\Delta_{1}+\Delta_{2}\right)\right)}{1+b U\left(\tau_{n}^{+}\right) / m(g+b-c) \bar{X}^{*}\left(\exp \left((g+b-c) \Delta_{1}\right)-1+(1-p) \exp \left((b-c) \Delta_{1}\right)\left(\exp \left((g+b-c) \Delta_{2}\right)\right)-1\right)}
$$

By induction, we can see that

$$
\begin{aligned}
U(t)= & \frac{(1-p)^{k} U\left(\tau_{n}^{+}\right) \exp \left(\sum_{i=1}^{k} \Delta_{i}\right) \exp \left((b-c)\left(t-t_{n}^{k}\right)\right)}{1+b U\left(\tau_{n}^{+}\right) / m(g+b-c) \bar{X}^{*}\left(\sum_{i=1}^{k}(1-p)^{(i-1)} \exp ((b-c)\right.}, \\
& \left.\sum_{j=1}^{i-1} \Delta_{j}\right)\left(\exp \left((g+b-c) \Delta_{i}\right)-1\right)+(1-p)^{k}\left(\exp \left((g+b-c)\left(t-t_{n}^{k}\right)\right)-1\right)
\end{aligned}
$$

for all $t \in\left(t_{n}^{k}, \tau_{n+1}\right]$. At time $\tau_{n+1}$, we only need to replant healthy plants once without removing infected plants.

$$
U\left(\tau_{n+1}^{+}\right)=\frac{(1-p)^{k} U\left(\tau_{n}^{+}\right) \exp ((b-c) \tau)}{1+b U\left(\tau_{n}^{+}\right) / m(g+b-c) \bar{X}^{*}\left(\sum_{i=1}^{k+1}(1-p)^{i-1} \exp \left((b-c) \sum_{j=1}^{i-1} \Delta_{j}\right)\left(\exp \left((g+b-c) \Delta_{i}\right)-1\right)\right.} .
$$

Denote $U_{\tau_{n}}=U\left(\tau_{n}^{+}\right)$; then, we have the following difference equation:

$$
U_{\tau_{n+1}}=\frac{(1-p)^{k} U_{\tau_{n}} \exp ((b-c) \tau)}{1+b U \tau_{n} / m(g+b-c) \bar{X}^{*} \sum_{i=1}^{k+1}(1-p)^{i-1} \exp \left((b-c) \sum_{j=1}^{i-1} \Delta_{j}\right)\left(\exp \left((g+b-c) \Delta_{i}\right)-1\right)} .
$$

Define $R_{3}=(1-p)^{k} \exp ((b-c) \tau)$, and we have the following: if $R_{3} \leq 1$, then the above difference equation always has a zeros equilibrium; if $R_{3}>1$, then it has a stable positive equilibrium:

$$
\bar{U}^{*}=\frac{(1-p)^{k} \exp ((b-c) \tau)-1}{b / m(g+b-c) \bar{X}^{*} \sum_{i=1}^{k+1}(1-p)^{i-1} \exp \left((b-c) \sum_{j=1}^{i-1} \Delta_{j}\right)\left(\exp \left((g+b-c) \Delta_{i}\right)-1\right)} .
$$

Thus, the system

$$
\begin{cases}\frac{\mathrm{d} U(t)}{\mathrm{d} t}=b U(t)\left(1-\frac{U(t)}{m X^{*}(t)}\right)-c U(t), & t \neq\left\{\tau_{n}, t_{n}^{i}\right\} \\ U\left(t^{+}\right)=(1-p) U(t), & t=t_{n}^{i}, \\ U\left(0^{+}\right)=U_{0} & \end{cases}
$$


has a positive periodic solution

$$
\bar{U}^{*}(t)=\left\{\begin{array}{l}
\frac{\bar{U}^{*} \exp \left((b-c)\left(t-\tau_{n}\right)\right)}{1+b \bar{U}^{*} / m(g+b-c) \bar{X}^{*}\left(\exp \left((g+b-c)\left(t-\tau_{n}\right)\right)-1\right)}, \quad t \in\left(\tau_{n}, t_{n}^{1}\right], \\
\frac{(1-p) \bar{U}^{*} t \operatorname{expn}\left((b-c) \Delta_{1}\right) q \operatorname{exph}\left((b-c)\left(t-t_{n}^{1}\right)\right)}{1+b \bar{U}^{*} / m(g+b-c) \bar{X}^{*}\left(\exp \left((g+b-c) \Delta_{1}\right)-1+(1-p) \exp \left((b-c) \Delta_{1}\right)\left(\exp \left((g+b-c)\left(t-t_{n}^{1}\right)\right)-1\right)\right)}, \quad t \in\left(t_{n}^{1}, t_{n}^{2}\right], \\
\vdots \\
\frac{(1-p)^{k} \bar{U}^{*} \exp \left((b-c) \sum_{i=1}^{k} \Delta_{i}\right) \exp \left((b-c)\left(t-t_{n}^{k}\right)\right)}{1+b \bar{U}^{*} / m(g+b-c) \bar{X}^{*}\left(\sum_{i=1}^{k}(1-p)^{(i-1)} \exp \left((b-c) \sum_{j=1}^{i-1} \Delta_{j}\right)\left(\exp \left((g+b-c) \Delta_{i}\right)-1\right)+(1-p)^{k} \exp \left((b-c) \sum_{i=1}^{k} \Delta_{i}\right)\left(\exp \left((g+b-c)\left(t-t_{n}^{k}\right)\right)-1\right)\right)}, t \in\left(t_{n}^{k}, \tau_{n+1}\right],
\end{array}\right.
$$

which indicates that we obtain the complete expression of periodic solution for system (92) over the time interval $\tau_{n}<t \leq \tau_{n+1}$.

Lemma 5. System (91) has two nonnegative periodic solutions $\left(\bar{X}^{*}(t), 0\right)$ and $\left(\bar{X}^{*}(t), \bar{U}^{*}(t)\right)$. Moreover, if $R_{3} \leq 1$, then for any solution $(X(t), U(t))$ of (91), we have $X(t) \longrightarrow \bar{X}^{*}(t), U(t) \longrightarrow 0$ as $t \longrightarrow \infty$; if $R_{3}>1$, then we have $X(t) \longrightarrow \bar{X}^{*}(t), U(t) \longrightarrow \bar{U}^{*}(t)$ as $t \longrightarrow \infty$.

Therefore, system (91) has a plant-only periodic solution $\left(\bar{X}^{*}(t), 0,0,0\right)$ and disease-free positive periodic solution $\left(\bar{X}^{*}(t), \bar{U}^{*}(t), 0,0\right)$. Due to the complexity of the model, it is possible but very complex to obtain the threshold conditions for the global stability of the above two periodic solutions. Therefore, we will use the numerical analysis method to discuss the influence of the control strategy on the stability of the above two boundary periodic solutions in the following.

\section{Numerical Investigations}

Due to the high dimension of the system, it is challenging to study the global dynamics of the system after integrating the pulse effect. Therefore, in this section, we employ the parameters given in Table 1 as the baseline parameters for more systematic numerical analysis. This can not only help to further determine the existing main conclusions, but also help to find new dynamic behaviors and reveal the key factors of plant disease control.

According to the main results related to model (1), if $b \leq c$ and $R_{01} \leq 1$, then the plant-only equilibrium $E_{01}$ is globally asymptotically stable; if $b>c$ and $R_{0} \leq 1$, then the disease-free equilibrium $E_{02}$ is globally asymptotically stable; and if $R_{0}>1$, then the disease is uniformly persistent. To confirm those results, we show the stability of the plant-only equilibrium of system (1) for $\alpha=0, b=0.1, c=0.18$ in Figure 2(a), and stability of the disease-free equilibrium of system (1) for $\alpha=0.02, b=0.2, c=0.12, R_{0}=0.7906<1$ in Figure 2(b). If we choose $\alpha=0, b=0.2, c=0.12$ with $R_{0}=1.9365>1$, then the plant disease is persistent, as shown in Figure 2(c). The effects of roguing rate $\alpha$ on the basic reproduction number $R_{0}$ are shown in Figure 3, from which we can see that $R_{0}$ is a strictly monotonic decreasing function of $\alpha$. This reveals that increasing the roguing rate can effectively reduce the threshold $R_{0}$, and then the disease could be successfully controlled.

In Figure 4(a), we show the stability of the plant-only periodic solution of model (25) with parameter set $\alpha=0.95, T=15, p=0.75$, and $R_{1}<1$; in Figure $4(\mathrm{~b})$, we show the stability of the disease-free periodic solution of model (25) with parameter set $\alpha=0.95, T=30$, $p=0.85, R_{1}<1$, and $R_{2}<1$. If we fix the roguing rate $\alpha=0.8$, $T=30$, and $p=0.6$, then $R_{1}>1$ and $R_{2}>1$, and then the numerical simulations reveal that the disease is persistent (Figure 4(c)). Figure 5 shows how different period $T$ and vectors removal rate $p$ affect the threshold value $R_{1}$. However, due to the complexity of model (25), we cannot get the analytical expression of the threshold $R_{2}$; thus, we use the methods proposed in $[39,40]$ to calculate the threshold value $R_{2}$. We fix the parameter set $T=30, p=0.85$, and $r=0.4$, and let the roguing rate $\alpha$ vary. It follows from Figure 6(a) that the threshold value $R_{2}$ is a monotonically decreasing function with respect to roguing rate $\alpha$. Similarly, for the parameter sets shown in Figure 6 , we see that $R_{2}$ is a monotonically decreasing function with respect to vectors removal rate $p$, as shown in Figure 6(b). On the other hand, $R_{2}$ is a monotonically increasing function with respect to impulsive period $T$ or replanting rate $r$, as shown in Figures 6(c) and 6(d), respectively. All those results indicate that increasing the roguing rate $\alpha$ and vectors removal rate $p$ and reducing the impulsive period $T$ and the replanting rate $r$ can effectively reduce the threshold $R_{2}$. Thus, it is conducive to the control of plant disease. Figure 7 further shows the effects of different parameter sets on the threshold condition $R_{2}$.

According to the theoretical analyses for model (91), we obtain two threshold values $R_{3}$ and $R_{\tau}^{k}$, and if $R_{3} \leq 1$, then the plant-only periodic solution $\left(\bar{X}^{*}(t), 0,0,0\right)$ is globally asymptotically stable. Indeed, in Figure $8(\mathrm{a})$, we capture the stability of the plant-only periodic solution $\left(\bar{X}^{*}(t), 0,0,0\right)$. In Figure 9, we fix the replanting period $\tau=60$ and let vectors removal rate $p=(0.95,0.9,0.75,0.65)$ vary. The simulation 

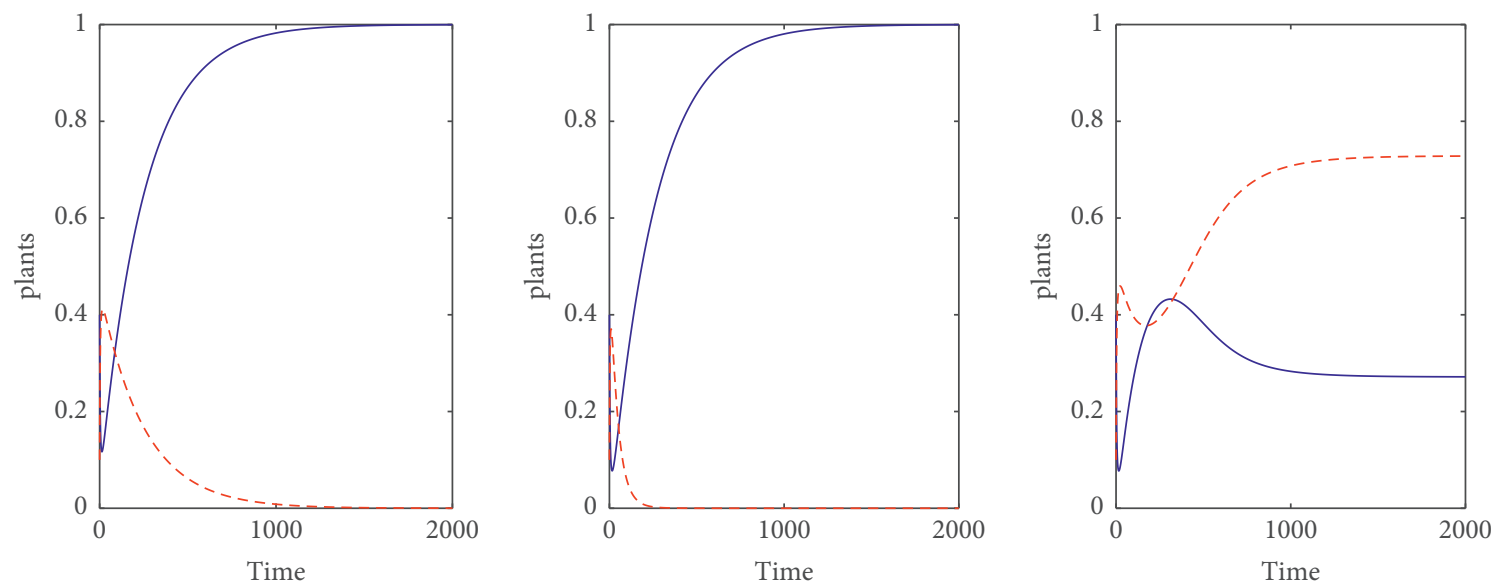

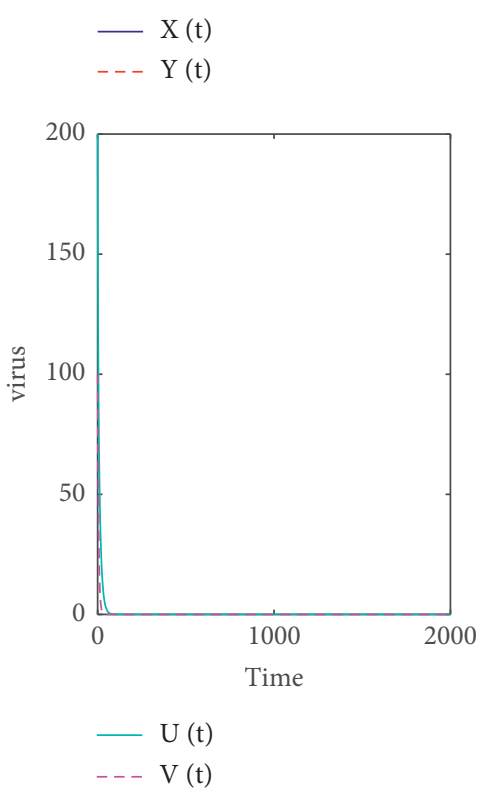

(a)

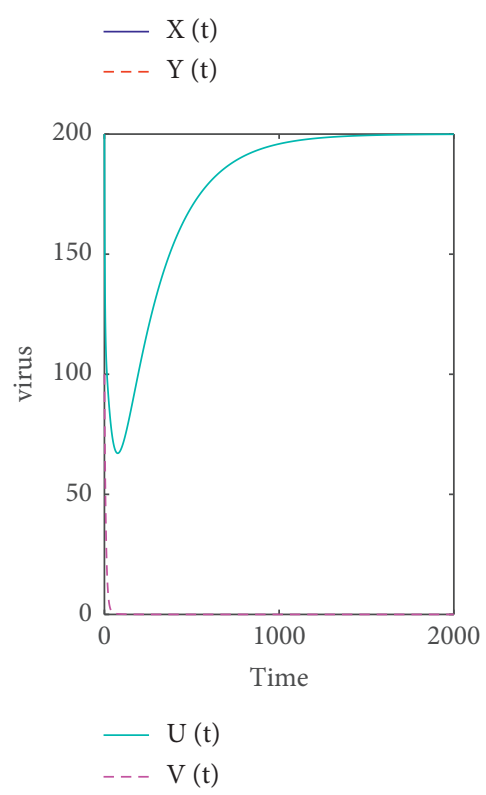

(b)
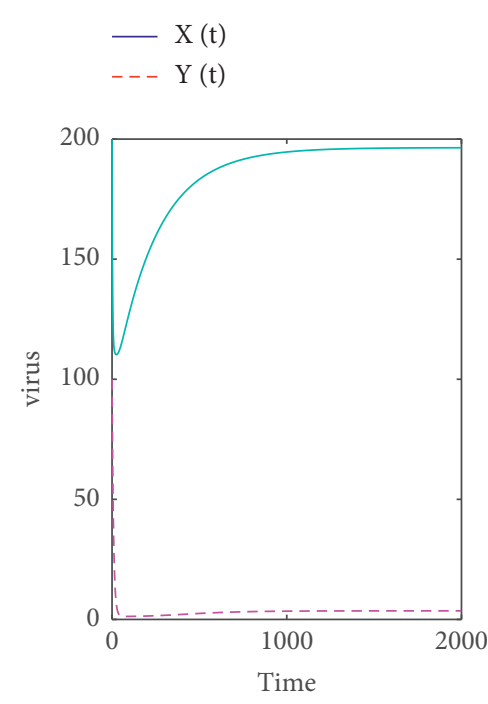

$-\mathrm{U}(\mathrm{t})$

(c)

Figure 2: Stabilities of the equilibria $E_{0 i}(i=1,2)$ and $E^{*}$ for model (1). The set of parameter values is as follows: $r=0.004, k_{1}=0.003, g=0.004, m=500, k_{2}=0.003$. (a) $\alpha=0, b=0.1, c=0.18$; (b) $\alpha=0.02, b=0.2, c=0.12, R_{0}=0.7906<1$; (c) $\alpha=0$, $b=0.2, c=0.12, R_{0}=1.9365>1$.

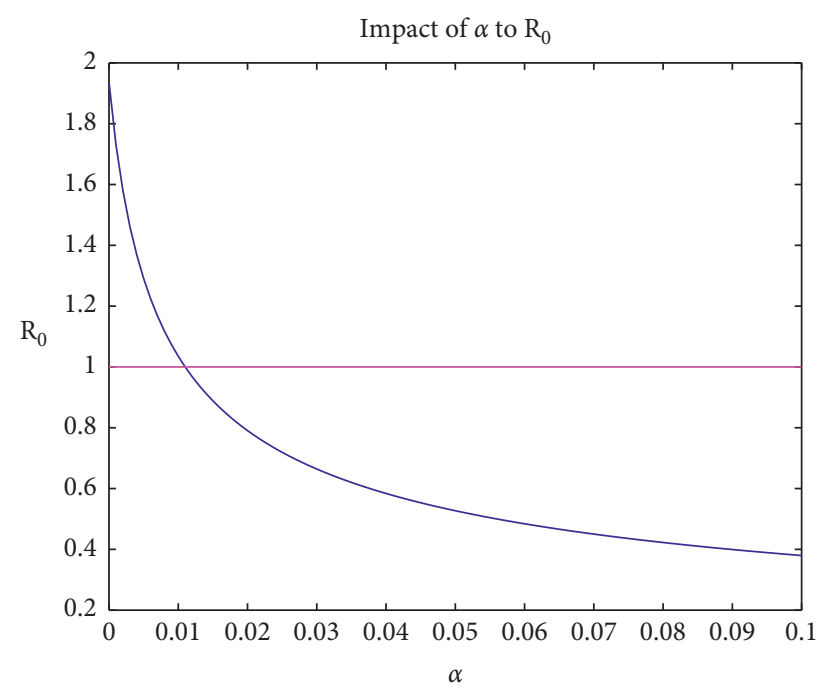

FIgURE 3: The effects of parameter $\alpha$ on the $R_{0}$. 

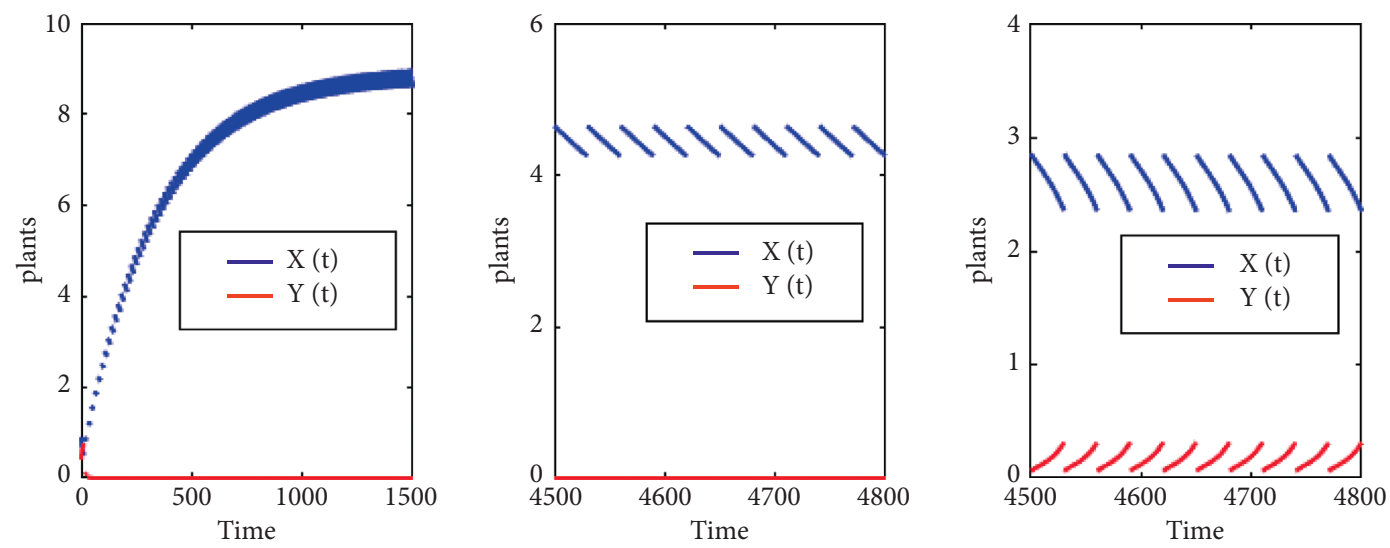

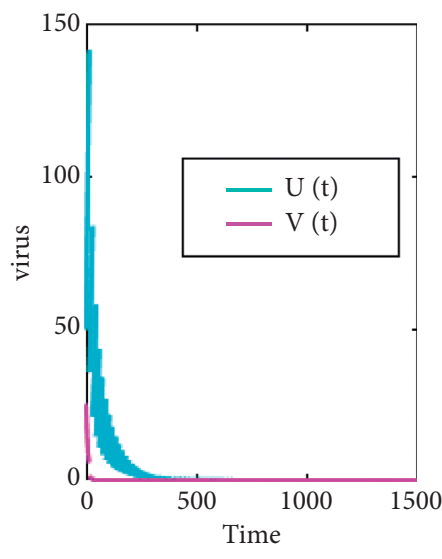

(a)

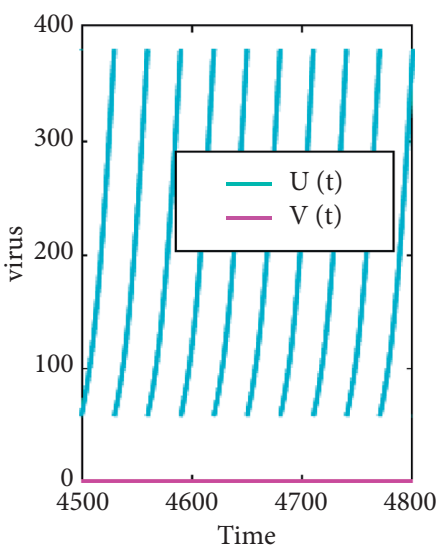

(b)

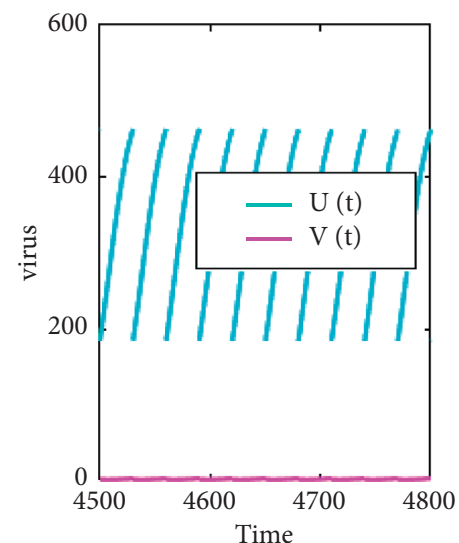

(c)

Figure 4: Existence and stability of periodic solutions for model (25). The set of parameter values is as follows: $r=0.4, k_{1}=0.003, g=0.003, b=0.2, m=500, k_{2}=0.003, c=0.12 . \quad(a) \quad T=15, p=0.75, \alpha=0.9, R_{1}=0.83<1 ; \quad(b) \quad T=30, p=0.85$, $\alpha=0.95, R_{1}=1.6535>1, R_{2}=0.9331<1$; (c) $T=30, p=0.6, \alpha=0.8, R_{1}=4.4093>1, R_{2}>1$.

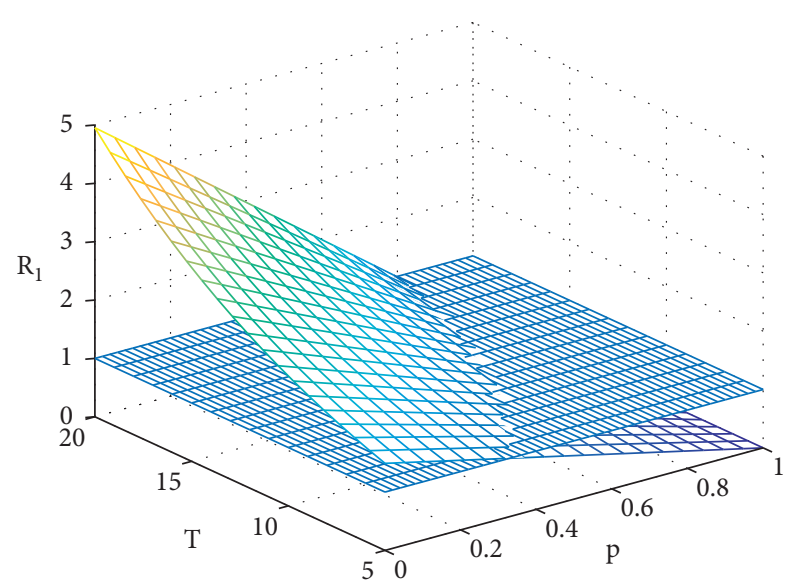

FIGURE 5: The effects of the parameter sets on the threshold values $R_{1}$.

results indicate that the times of roguing control $k$ is inversely proportional to vectors removal rate $p$. In order to maintain the threshold value $R_{3}$ less than one, we find that from Figure 9 the times of roguing control $k$ should be increased significantly as vectors removal rate $p$ is decreasing. Figure 10 further shows the effects of different parameter sets on the threshold condition $R_{3}$. Note that the value of $R_{3}$ appears to be quite sensitive to small changes in removal rate $p$, times of roguing control $k$, and replanting rate $r$.

In order to discuss the influence of the control strategies on the stability of the disease-free positive periodic solution $\left(\bar{X}^{*}(t), \bar{U}^{*}(t), 0,0\right)$, similar to the calculation of $R_{2}$, we can obtain a threshold $R_{\tau}^{k}=\rho\left(H_{2}^{k} \Phi_{\left(\bar{F}(t)-V_{r}(t)\right)}(\tau)\right)$; here, $V_{r}(t)$ and $H_{2}$ can be seen in (56) and (58).

$$
\bar{F}(t)=\left(\begin{array}{cc}
0 & k_{1} \bar{X}^{*}(t) \\
k_{2} \bar{U}^{*}(t) & 0
\end{array}\right) .
$$

Due to the complexity of model (90) and the inability to calculate the exact expression of $R_{\tau}^{k}$, we let the times of roguing control $k$ vary and fix the other parameters. The numerical results show that if $k=3$ and $R_{3}>1$, then the threshold value $R_{\tau}^{k}<1$, which indicates that the disease-free periodic solution is globally asymptotically stable (Figure $8(\mathrm{~b})$ ). If $k=1,2$ and $R_{3}>1$, then the threshold value $R_{\tau}^{k}>1$, which indicates that the endemic periodic solution is persistent (Figure 8(c)).

Therefore, the optimal control of plant diseases is in fact to choose suitable times of roguing control $k$ such that $R_{\tau}^{k}<1$ and $R_{\tau}^{k-1}>1$. In Figure 11, we fix the replanting period $\tau$ and 


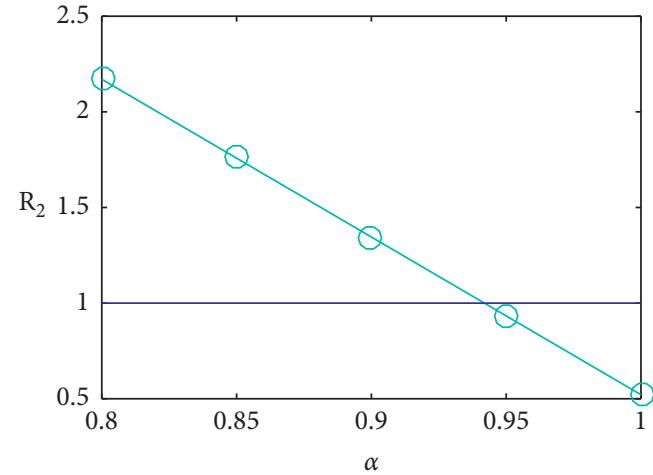

(a)

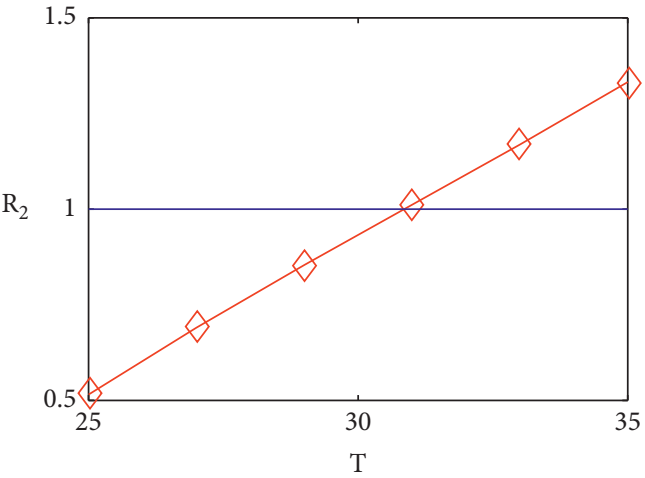

(c)

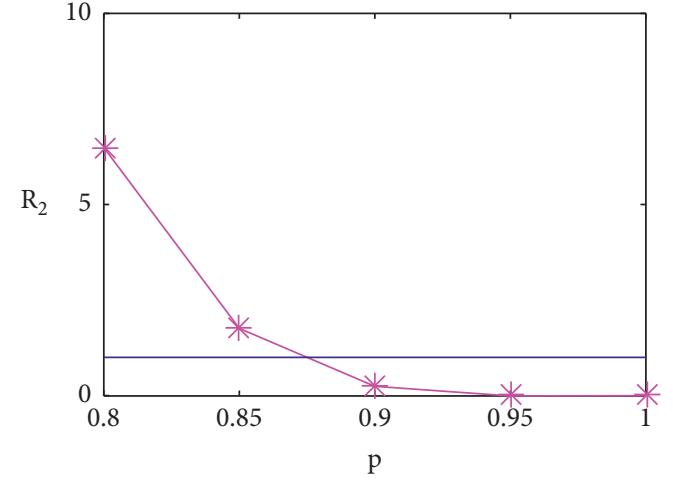

(b)

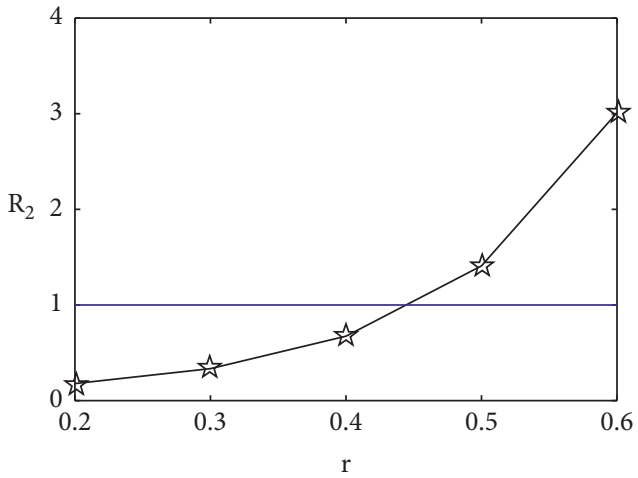

(d)

Figure 6: The effects of the parameter sets on the threshold level $R_{2}$. The set of parameter values is as follows: $b=0.2, c=0.12, m=500, k_{1}=0.003, k_{2}=0.003, g=0.003$. (a) $T=30, p=0.85, r=0.4$; (b) $T=30, \alpha=0.85, r=0.4$; (c) $p=0.82, \alpha=0.82$, $r=0.4$; (d) $T=30, \alpha=0.9, p=0.87$.

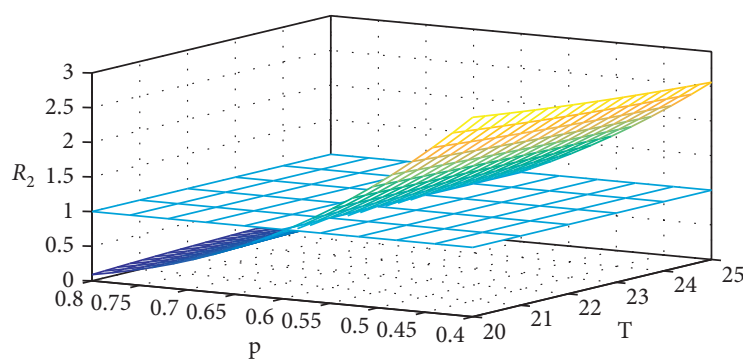

(a)

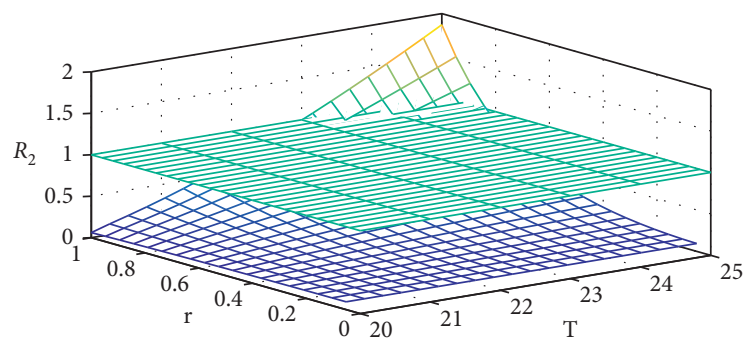

(c)

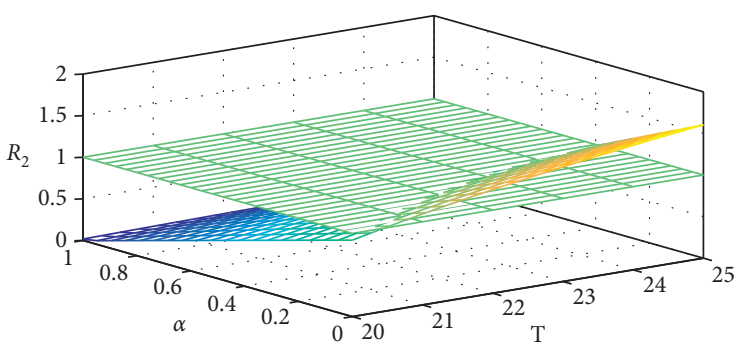

(b)

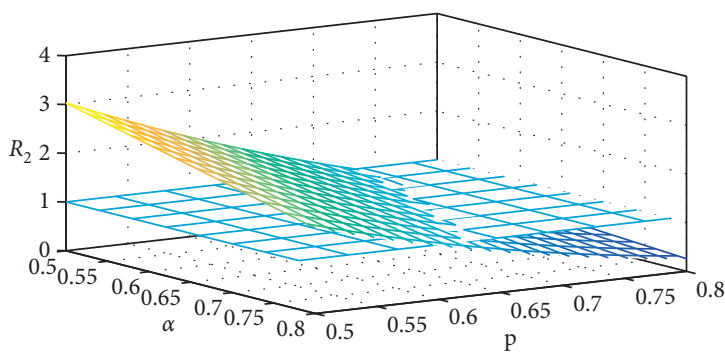

(d)

Figure 7: The effects of the parameter sets on the threshold level $R_{2}$. The set of parameter values is $b=0.2, c=0.12, m=500$, $k_{1}=0.003, k_{2}=0.003, g=0.003$. (a) $r=0.4, \alpha=0.9$; (b) $r=0.4, p=0.8$; (c) $p=0.8, \alpha=0.9$; (d) $r=0.3, T=22$. 

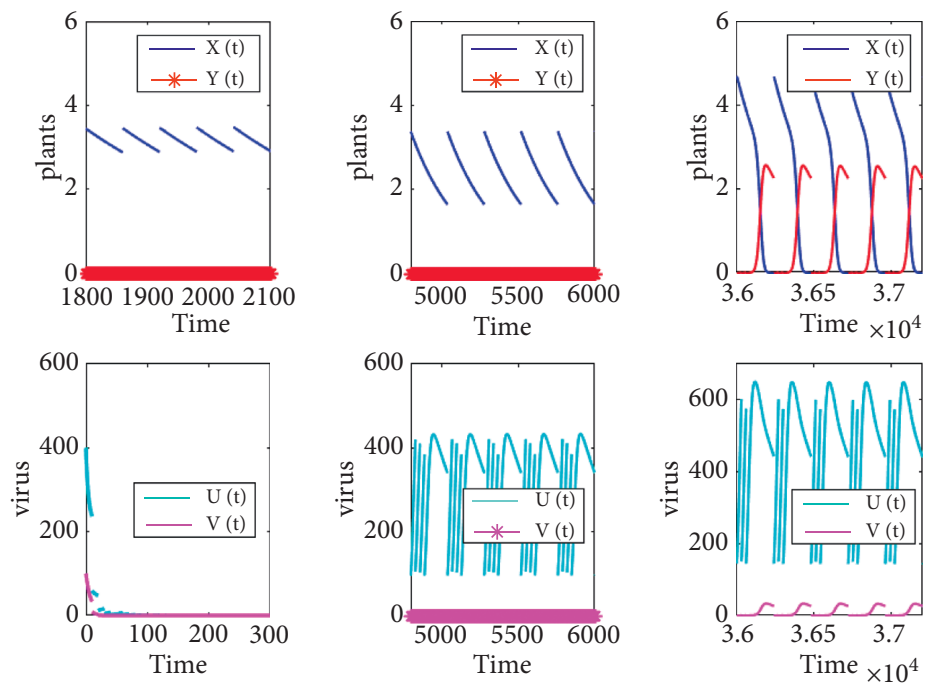

(a)

(b)

(c)

Figure 8: The existence and stability of the periodic solution $\left(\bar{X}^{*}(t), 0,0,0\right)$ of model $(90)$. The set of parameter values is as follows: $k_{1}=0.003, g=0.003, b=0.2, m=500, k_{2}=0.003, c=0.12$. (a) $\tau=60, \Delta_{i}=\Delta=10, p=0.75, r=0.4, \alpha=0.9, k=4, R_{3} \leq 1$; (b) $\tau=240$, $\Delta_{i}=\Delta=30, p=0.75, r=0.8, \alpha=0.95, k=3, R_{3}>1, R_{\tau}^{k}<1$; (c) $\tau=240, \Delta_{i}=\Delta=30, p=0.75, r=0.8, \alpha=0.95, b=0.2, k=2, R_{3}>1 R_{\tau}^{k}>1$.

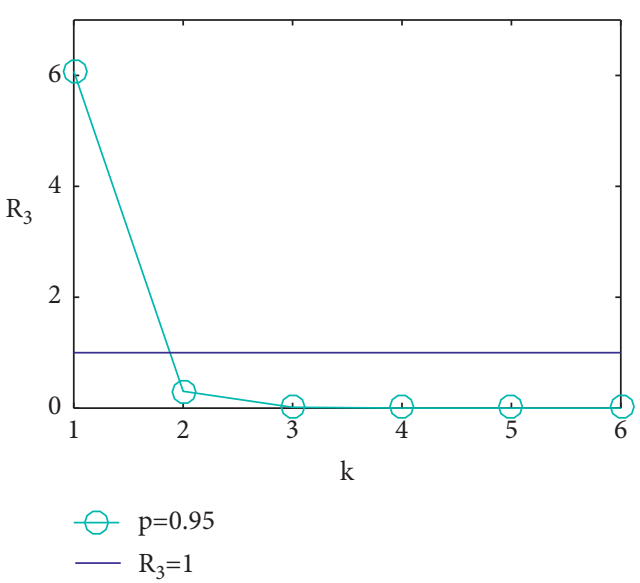

(a)

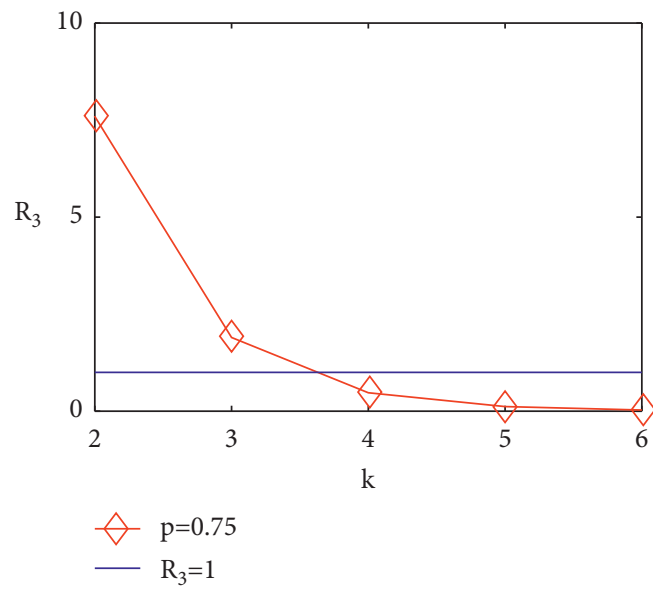

(c)

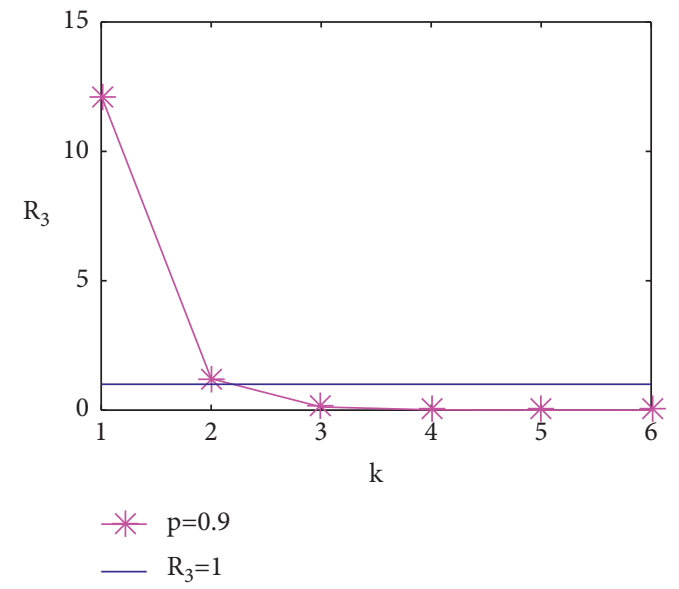

(b)

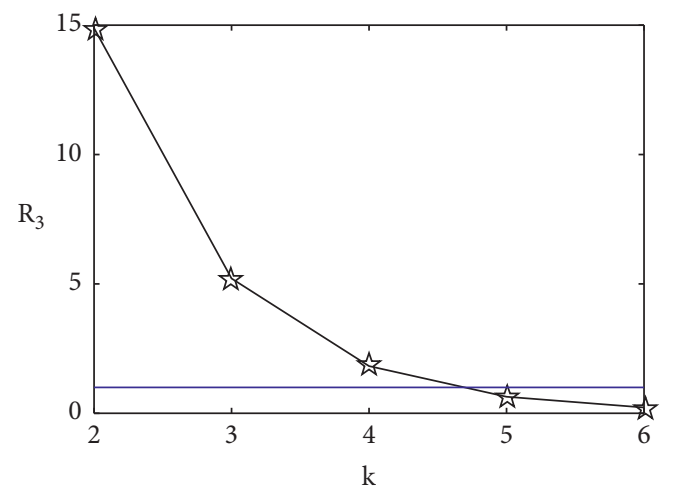

$\mathrm{p}=0.65$
$\mathrm{R}$
$\mathrm{R}_{3}=1$

Figure 9: The effects of the times of roguing control $k$ and the vectors removal rate $p$ on the threshold level $R_{3}$. The set of parameter values is as follows: $\tau=60, b=0.2, c=0.12$, and $k=1,2,3, \ldots, 6$. (a) $p=0.95$; (b) $p=0.9$; (c) $p=0.75$; (d) $p=0.65$. 


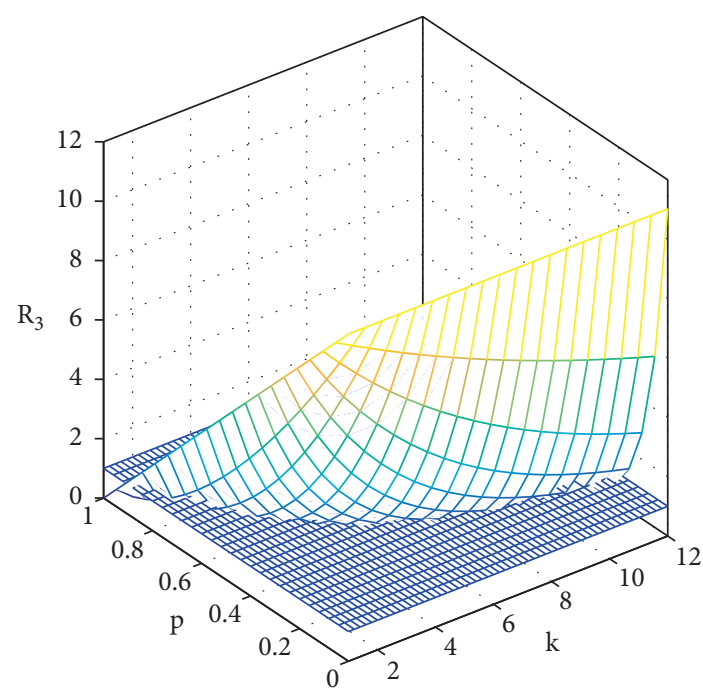

(a)

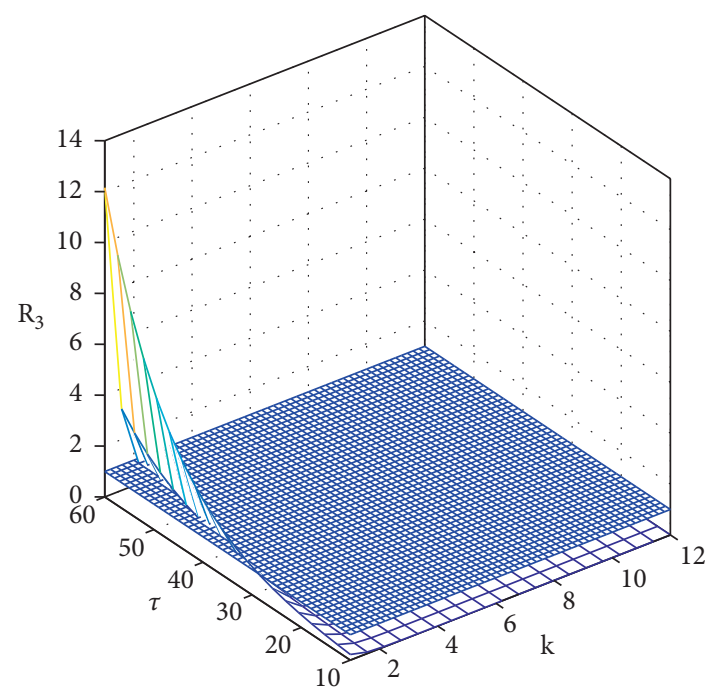

(b)

FIGURE 10: The effects of the times of roguing control $k$ and parameter sets on the threshold level $R_{3}$. The set of parameter values is as follows: $b=0.2, c=0.12$, and $k=1,2,3, \ldots, 6$. (a) $\tau=30$; (b) $p=0.9$.
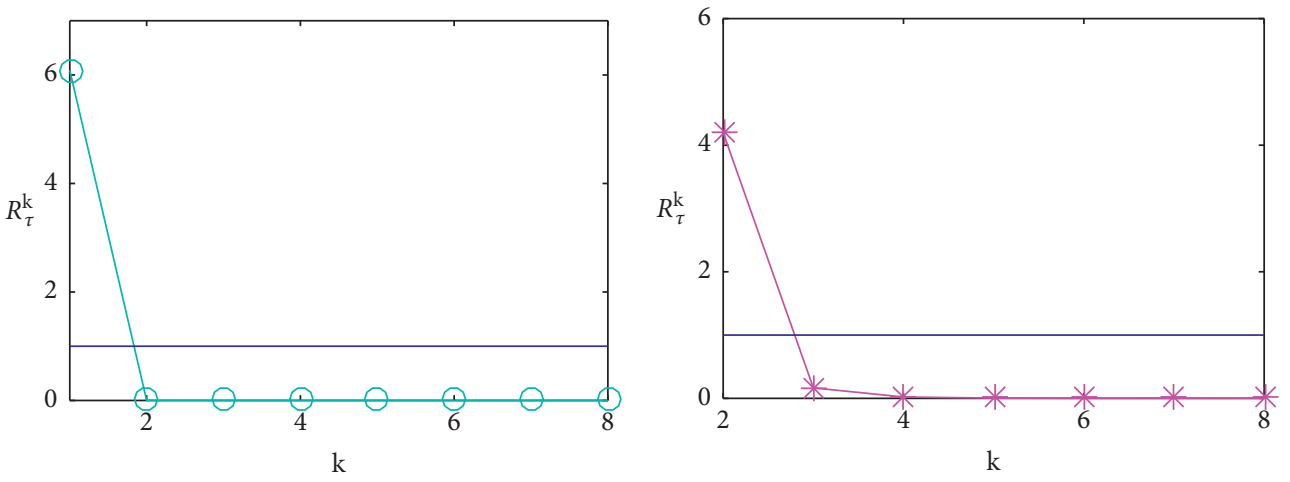

$$
\begin{aligned}
& \bigcirc \mathrm{p}=0.99 \\
& -R_{\tau}^{\mathrm{k}}=1
\end{aligned}
$$

(a)

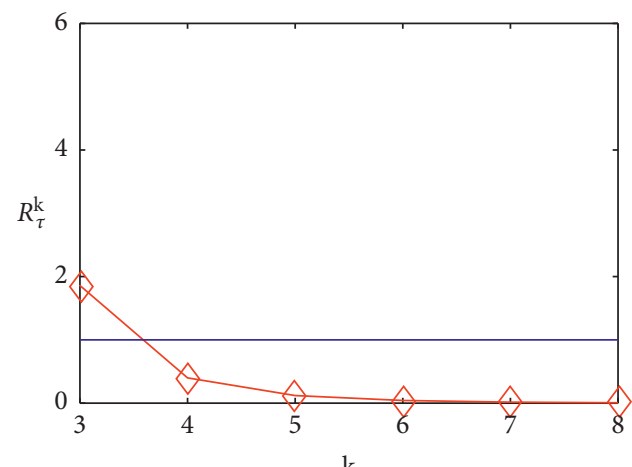

$\mathrm{k}$

$$
\begin{aligned}
& \vartheta \mathrm{p}=0.5 \\
& -R_{\tau}^{\mathrm{k}}=1
\end{aligned}
$$

(c)

$$
\begin{aligned}
& \text { * } \mathrm{p}=0.7 \\
& -R_{\tau}^{\mathrm{k}}=1
\end{aligned}
$$

(b)

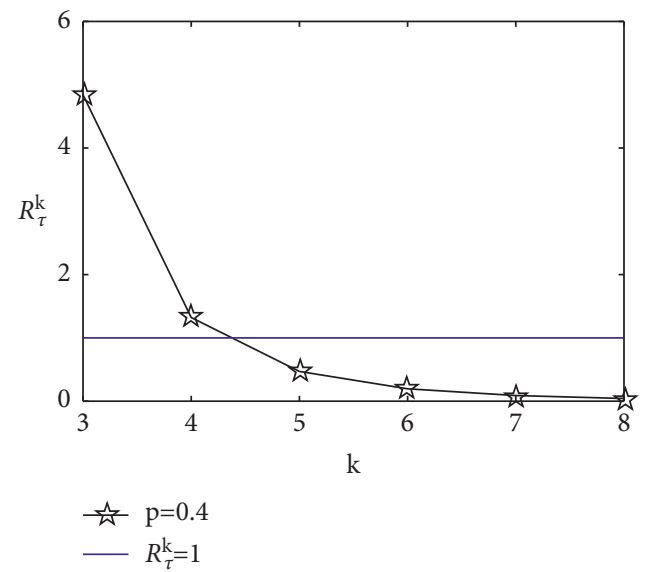

(d)

FIGURE 11: The effects of the times of roguing control $k$ and the vectors removal rate $p$ on the threshold level $R_{\tau}^{k}$. The set of parameter values is as follows: $b=0.2, c=0.12, m=500, k_{1}=0.003, k_{2}=0.003, g=0.003, r=0.8, \tau=240, \Delta_{i}=\Delta=30$, and $k=1,2,3, \ldots, 8$. (a) $\alpha=0.99$, $p=0.99$; (b) $\alpha=0.95, p=0.7$; (c) $\alpha=0.95, p=0.5$; (d) $\alpha=0.95, p=0.4$. 


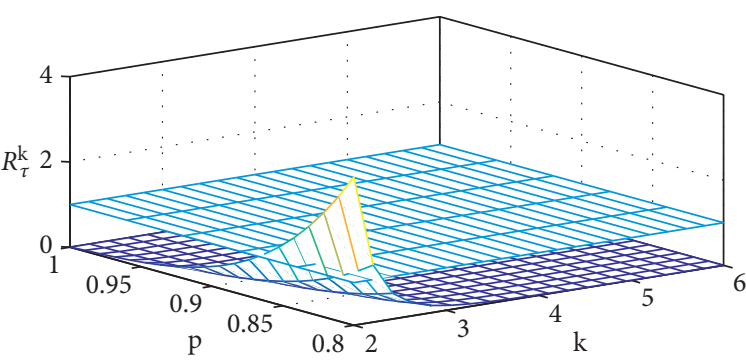

(a)

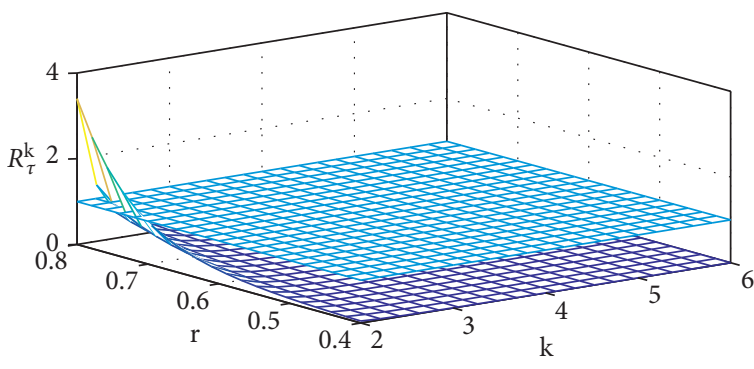

(c)

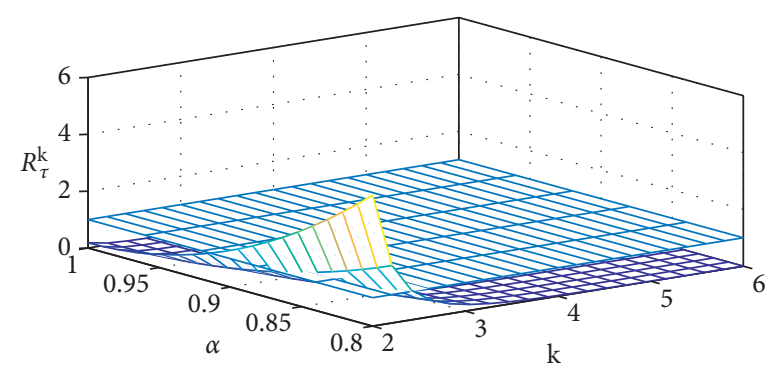

(b)

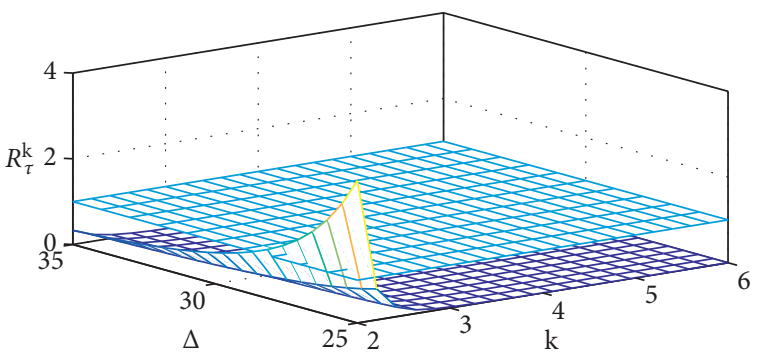

(d)

FIGURE 12: The effects of the times of roguing control $k$ and parameter sets on the threshold level $R_{\tau}^{k}$. The set of parameter values is as follows: $b=0.2, c=0.12, k_{1}=0.003, k_{2}=0.003, g=0.003, \tau=240, \quad$ and $\quad k=1,2,3, \ldots, 6 . \quad\left(\right.$ a) $\quad \alpha=0.95, \Delta_{i}=\Delta=30, r=0.8 ; \quad(b) \quad p=0.9$, $\Delta_{i}=\Delta=30, r=0.8$; (c) $\alpha=0.87, p=0.87, \Delta_{i}=\Delta=30$; (d) $\alpha=0.9, p=0.9, r=0.8, \Delta_{i}=\Delta$.

the removal period $\Delta_{i}=\Delta$ and let roguing rate $\alpha$ and the vectors removal rate $p$ vary. The simulation results indicate that the vectors removal rate can affect the times of controls when the infected plants are removed. This shows that the vectors removal is important when removing infected plants. Figure 12 shows the effects of different parameter sets on the threshold condition $R_{\tau}^{k}$. All simulation results shown in Figure 12 indicate that $R_{\tau}^{k}$ appears to be quite sensitive to small changes in roguing rate $\alpha$ and vectors removal rate $p$, replanting rate $r$, and removal period $\Delta$.

\section{Discussion}

Based on the characteristics of plant diseases transmitted by insect vectors $[7,8]$, we proposed the mathematical models with continuous control strategy and pulse control strategy with single-pulse sequence of multipulse sequences, developed the corresponding threshold theoretical analysis and numerical techniques to address the threshold dynamics of the model, and obtained the corresponding threshold conditions [10].

The main results for the model with continuous removing of infected plants reveal that we can either control the death rate of vector population (i.e., $b<c$ here) such that the plant-only equilibrium is globally asymptotically stable, or control the plant roguing rate such that the basic reproductive number $R_{0}$ is less than one, and consequently the plant disease-free equilibrium is globally asymptotically stable. All those indicate that the infected plants and infected vectors could be completely eradicated if we implement the removal control strategies properly.

According to the fact that infected plants are removed and some vectors are removed at the same time, we develop a pulse control model with fixed moments, which allows us to choose the period of impulsive control strategies such that the plant-only periodic solution and plant diseasefree periodic solution are globally asymptotically stable. Based on the above purpose, we employ the relevant theory of impulsive differential equations to address the threshold dynamics of the proposed model, and the threshold values which guarantee the global stability of plant-only and plant disease-free periodic solutions have been given. The threshold conditions show that the infected plants and infected vectors can be completely eradicated if we implement the cultural control strategies relatively frequently.

When considering the strategy of removing infected plants multiple times in a replanting cycle, a more realistic model has been proposed, which allows us to analyze and design the optimal control strategy such that the threshold value reaches its minimum. The optimal times of roguing infected plants within a replanting cycle can be numerically calculated, which can help us to design and optimize the prevention and control strategy of plant virus transmission. Finally, various numerical investigations have been given to confirm the main conclusions and reveal the optimal control strategies, and the biological implications of the main results have been briefly discussed in this section.

As we all know, insect vectors of plant diseases live directly on the corresponding infected plants. Therefore, when the corresponding infected plants or even healthy plants are removed, a large number of insect vectors will inevitably be removed. The above factors are undoubtedly beneficial to the prevention and control of plant virus disease transmission, but how to formulate and describe the above factors, develop corresponding mathematical models, and 
carry out systematic analysis is challenging, which is undoubtedly one of our important work in a later stage.

\section{Data Availability}

There were no data used to support this study.

\section{Conflicts of Interest}

The authors declare that they have no conflicts of interest regarding the publication of this paper.

\section{Acknowledgments}

This work was supported by the National Natural Science Foundation of China (NSFC) (12031010, 11631012, and 61772017).

\section{References}

[1] R. Marcus, S. Fishman, H. Talpaz, R. Salomon, and M. BarJoseph, "On the spatial distribution of citrus tristeza virus disease," Phytoparasitica, vol. 12, no. 1, pp. 45-52, 1984.

[2] J. Bové, "Huanglongbing: a destructive, newly-emerging, century-old disease of citrus," Journal of Plant Pathology, vol. 88 , no. 1, pp. 7-37, 2006.

[3] Y. Dumont and F. Chiroleu, "Vector control for the chikungunya disease," Mathematical Biosciences and Engineering, vol. 7, no. 2, pp. 313-345, 2010.

[4] M. Aranzazu and A. Fereres, "Virus diseases in lettuce in the mediterranean basin," Advances in Virus Research, vol. 84, pp. 247-288, 2012.

[5] G. Otim-Nape, A. Bua, and J. Thresh, The Current Pandemic of Cassava Mosaic Virus Disease in East Africa and its Control, Natural Resources Institute, Chatham, UK, 2000.

[6] F. van den Bosch, G. Akudibilah, S. Seal, and M. Jeger, "Host resistance and the evolutionary response of plant viruses," Journal of Applied Ecology, vol. 43, no. 3, pp. 506-516, 2006.

[7] M. J. Jeger, J. Holt, F. van den Bosch, and L. V. Madden, "Epidemiology of insect-transmitted plant viruses: modelling disease dynamics and control interventions," Physiological Entomology, vol. 29, no. 3, pp. 291-304, 2004.

[8] M. Jeger, F. van den Bosch, L. Madden, and J. Holt, "A model for analysing plant-virus transmission characteristics and epidemic development," Mathematical Medicine and Biology, vol. 15, no. 1, pp. 1-18, 1998.

[9] Y. Dumont and J. M. Tchuenche, "Mathematical studies on the sterile insect technique for the chikungunya disease and aedes albopictus," Journal of Mathematical Biology, vol. 65, no. 5, pp. 809-854, 2012.

[10] M. D. Asfaw, S. M. Kassa, and E. M. Lungu, "Stochastic plantherbivore interaction model with allee effect," Journal of Mathematical Biology, vol. 79, no. 6, pp. 2183-2209, 2019.

[11] R. Shi, H. Zhao, and S. Tang, "Global dynamic analysis of a vector-borne plant disease model," Advances in Difference Equations, vol. 2014, no. 1, p. 59, 2014.

[12] S. Fishman and R. Marcus, "A model for spread of plant disease with periodic removals," Journal of Mathematical Biology, vol. 21, no. 2, pp. 149-158, 1984.

[13] S. Tang and R. A. Cheke, "Models for integrated pest control and their biological implications," Mathematical Biosciences, vol. 215, no. 1, pp. 115-125, 2008.
[14] F. van den Bosch, M. J. Jeger, and C. A. Gilligan, "Disease control and its selection for damaging plant virus strains in vegetatively propagated staple food crops; a theoretical assessment," Proceedings of the Royal Society B: Biological Sciences, vol. 274, no. 1606, pp. 11-18, 2007.

[15] M. J. Jeger, A. J. Termorshuizen, M. P. M. Nagtzaam, and F. van den Bosch, "The effect of spatial distributions of mycoparasites on biocontrol efficacy: a modelling approach," Biocontrol Science and Technology, vol. 14, no. 4, pp. 359-373, 2004.

[16] S. Tang, J. Liang, Y. Tan, and R. A. Cheke, "Threshold conditions for integrated pest management models with pesticides that have residual effects," Journal of Mathematical Biology, vol. 66, no. 1, pp. 1-35, 2013.

[17] S. Tang, Y. Xiao, and R. A. Cheke, "Dynamical analysis of plant disease models with cultural control strategies and economic thresholds," Mathematics and Computers in Simulation, vol. 80, no. 5, pp. 894-921, 2010.

[18] T. Zhao, Y. Xiao, and R. J. Smith, "Non-smooth plant disease models with economic thresholds," Mathematical Biosciences, vol. 241, no. 1, pp. 34-48, 2013.

[19] T. Zhao and Y. Xiao, "Plant disease models with nonlinear impulsive cultural control strategies for vegetatively propagated plants," Mathematics and Computers in Simulation, vol. 107pp. 61-91, C, 2015.

[20] T. Zhao and R. J. Smith, "Global dynamical analysis of plantdisease models with nonlinear impulsive cultural control strategy," Mathematical Biosciences and Engineering, vol. 16, no. 6, pp. 7022-7056, 2019.

[21] J. Holt, M. J. Jeger, J. M. Thresh, and G. W. Otim-Nape, “An epidemilogical model incorporating vector population dynamics applied to African Cassava mosaic virus disease," Journal of Applied Ecology, vol. 34, no. 3, pp. 793-806, 1997.

[22] L. Luo, S. Gao, Y. Ge, and Y. Luo, "Transmission dynamics of a Huanglongbing model with cross protection," Advances in Difference Equations, vol. 2017, no. 1, p. 355, 2017.

[23] S. Tang, Y. Xiao, L. Chen, and R. Cheke, "Integrated pest management models and their dynamical behaviour," Bulletin of Mathematical Biology, vol. 67, no. 1, pp. 115-135, 2005.

[24] A. K. Shaw, M. Igoe, A. G. Power, N. A. Bosque-Pérez, and A. Peace, "Modeling approach influences dynamics of a vector-borne pathogen system," Bulletin of Mathematical Biology, vol. 81, no. 6, pp. 2011-2028, 2019.

[25] F. Al Basir, S. Adhurya, M. Banerjee, E. Venturino, and S. Ray, "Modelling the effect of incubation and latent periods on the dynamics of vector-borne plant viral diseases," Bulletin of Mathematical Biology, vol. 82, no. 7, p. 94, 2020.

[26] M. J. Jeger, Z. Chen, G. Powell, S. Hodge, and F. van den Bosch, "Interactions in a host plant-virus-vectorparasitoid system: modelling the consequences for virus transmission and disease dynamics," Virus Research, vol. 159, no. 2, pp. 183-193, 2011.

[27] F. A. Basir, E. Venturino, S. Ray, and P. K. Roy, "Impact of farming awareness and delay on the dynamics of mosaic disease in jatropha curcas plantations," Computational and Applied Mathematics, vol. 37, no. 5, pp. 6108-6131, 2018.

[28] S. Tang and R. A. Cheke, "State-dependent impulsive models of integrated pest management (IPM) strategies and their dynamic consequences," Journal of Mathematical Biology, vol. 50, no. 3, pp. 257-292, 2005.

[29] Y. Pei, X. Ji, and C. Li, "Pest regulation by means of continuous and impulsive nonlinear controls," Mathematical and Computer Modelling, vol. 51, no. 5-6, pp. 810-822, 2010. 
[30] S. Tang and R. A. Cheke, "Models for integrated pest control and their biological implications," Mathematical Biosciences, vol. 215, no. 1, pp. 115-125, 2008.

[31] S. Tang and Y. Xiao, "One-compartment model with michaelis-menten elimination kinetics and therapeutic window: an analytical approach," Journal of Pharmacokinetics and Pharmacodynamics, vol. 34, no. 6, pp. 807-827, 2007.

[32] X. Meng and Z. Li, "The dynamics of plant disease models with continuous and impulsive cultural control strategies," Journal of Theoretical Biology, vol. 266, no. 1, pp. 29-40, 2010.

[33] S. Gao, L. Xia, Y. Liu, and D. Xie, "A plant virus disease model with periodic environment and pulse roguing," Studies in Applied Mathematics, vol. 136, no. 4, pp. 357-381, 2010.

[34] N. Rakshit, F. Al Basir, A. Banerjee, and S. Ray, "Dynamics of plant mosaic disease propagation and the usefulness of roguing as an alternative biological control," Ecological Complexity, vol. 38, pp. 15-23, 2019.

[35] P. van den Driessche and J. Watmough, "Reproduction numbers and sub-threshold endemic equilibria for compartmental models of disease transmission," Mathematical Biosciences, vol. 180, no. 1-2, pp. 29-48, 2002.

[36] J. LaSalle, The Stability of Dynamical Systems, Regional Conference Series in Applied Mathematics, SIAM, Philadelphia, PA, USA, 1976.

[37] H. I. Freedman, S. Ruan, and M. Tang, "Uniform persistence and flows near a closed positively invariant set," Journal of Dynamics and Differential Equations, vol. 6, no. 4, pp. 583600, 1994.

[38] D. Bainov and P. Simeonov, Impulsive Differential Equations: Periodic Solutions and Applications, Longman Scientific and Technical, New York, NY, USA, 1993.

[39] Y. Yang and Y. Xiao, "The effects of population dispersal and pulse vaccination on disease control," Mathematical and Computer Modelling, vol. 52, no. 9-10, pp. 1591-1604, 2010.

[40] Y. Yang and Y. Xiao, "Threshold dynamics for compartmental epidemic models with impulses," Nonlinear Analysis: Real World Applications, vol. 13, no. 1, pp. 224-234, 2012.

[41] W. Walter, Ordinary Differential Equations, Springer-Verlag, New York, NY, USA, 1998. 